\title{
Reproduction of Exponential Polynomials by Multivariate Non-stationary Subdivision Schemes with a General Dilation Matrix
}

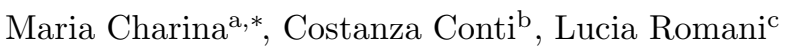 \\ ${ }^{a}$ Fakultät für Mathematik, TU Dortmund, D-44221 Dortmund, Germany \\ ${ }^{b}$ Dipartimento di Energetica, Università di Firenze, Via C. Lombroso 6/17, I-50134 Firenze \\ ${ }^{c}$ Dipartimento di Matematica e Applicazioni, Università di Milano-Bicocca, Via R. Cozzi \\ 53, 20125 Milano, Italy
}

\begin{abstract}
We study scalar multivariate non-stationary subdivision schemes with a general dilation matrix. We characterize the capability of such schemes to reproduce exponential polynomials in terms of simple algebraic conditions on their symbols. These algebraic conditions provide a useful theoretical tool for checking the reproduction properties of existing schemes and for constructing new schemes with desired reproduction capabilities and other enhanced properties. We illustrate our results with several examples.

Keywords: Non-stationary subdivision schemes, reproduction and generation of exponential polynomials, algebraic conditions on subdivision symbols, subdivision parametrization
\end{abstract}

\section{Introduction}

In this paper we study multivariate non-stationary subdivision schemes which are iterative algorithms based on the repeated application of subdivision operators

$$
S_{\mathbf{a}^{[k]}}: \ell\left(\mathbb{Z}^{s}\right) \rightarrow \ell\left(\mathbb{Z}^{s}\right), \quad \mathbf{f}^{[k+1]}=S_{\mathbf{a}^{[k]}} \mathbf{f}^{[k]}, \quad k \geq 0 .
$$

The linear subdivision operators $S_{\mathbf{a}^{[k]}}$

$$
\left(S_{\mathbf{a}^{[k]}} \mathbf{f}^{[k]}\right)_{\boldsymbol{\alpha}}=\sum_{\boldsymbol{\beta} \in \mathbb{Z}^{s}} \mathrm{a}_{\boldsymbol{\alpha}-M \boldsymbol{\beta}}^{[k]} f_{\boldsymbol{\beta}}^{[k]}, \quad \boldsymbol{\alpha} \in \mathbb{Z}^{s}, \quad k \geq 0 .
$$

depend on a dilation matrix $M \in \mathbb{Z}^{s \times s}$ and on the finite sequences of real numbers $\mathbf{a}^{[k]}=\left\{\mathrm{a}_{\boldsymbol{\alpha}}^{[k]}, \boldsymbol{\alpha} \in \mathbb{Z}^{s}\right\}$. If convergent, subdivision schemes are used e.g. for designing curves, surfaces, or multivariate functions.

\footnotetext{
${ }^{*}$ Corresponding author

Email addresses: maria.charina@uni-dortmund.de (Maria Charina), costanza.conti@unifi.it (Costanza Conti), lucia.romani@unimib.it (Lucia Romani)
} 
The generation properties of subdivision schemes are well-understood and are characterized in terms of so-called zero conditions, see e.g. [6, 18, 26], on the mask symbols

$$
a^{[k]}(\boldsymbol{z})=\sum_{\boldsymbol{\alpha} \in \mathbb{Z}^{s}} \mathrm{a}_{\boldsymbol{\alpha}}^{[k]} \boldsymbol{z}^{\boldsymbol{\alpha}}, \quad k \geq 0 \quad \boldsymbol{z} \in(\mathbb{C} \backslash\{0\})^{s} .
$$

These conditions on the symbols determine if the subdivision limit belongs to the function space

$$
E P_{\Gamma, \Lambda}=\operatorname{span}\left\{\boldsymbol{x}^{\gamma} e^{\boldsymbol{\lambda} \cdot \boldsymbol{x}}: \boldsymbol{\gamma} \in \Gamma, \boldsymbol{\lambda} \in \Lambda\right\}, \quad \Gamma \subset \mathbb{N}_{0}^{s}, \quad \Lambda \subset \mathbb{C}^{s},
$$

if the starting sequence $\mathbf{f}^{[0]}$ in (1.1) is sampled from a function in this space. If for all functions in $E P_{\Gamma, \Lambda}$, the subdivision scheme generates exactly the same function from which the corresponding starting sequence $\mathbf{f}^{[0]}$ is sampled, then we say that the scheme reproduces $E P_{\Gamma, \Lambda}$.

The main goal of our study is to characterize the reproduction property of subdivision in terms of zero conditions and some additional conditions on subdivision symbols. These additional algebraic conditions provide a useful theoretical tool that simplifies the analysis of reproduction properties of existing schemes and the construction of new schemes with desired reproduction properties. We would like to emphasize that the properties of non-stationary subdivision schemes could be characterized in the Fourier domain in terms of the properties of the associated basic refinable functions, see e.g [5, 19, 22, 27, 33, 34]. Nevertheless, we think that it is more advantageous, for construction of new non-stationary schemes, to provide such characterizations in terms of simple algebraic conditions on the coefficients $\mathbf{a}^{[k]}, k \geq 0$, of the corresponding refinement equations. The reproduction and generation of $E P_{\Gamma, \Lambda}$ or its subspaces are crucial for modeling objects of different shapes that e.g. are described by polynomial, trigonometric or hyperbolic functions. Thus, these reproduction and generation properties of subdivision are important in CAGD, motion planning, iso-geometric analysis and for studying approximation properties of subdivision schemes [30, 24]. In spite of their importance, reproduction properties of non-stationary subdivision schemes have not been yet studied as rigorously as it is done in the stationary case when the mask a does not depend on the level $k$ of subdivision recursion. In the stationary case, characterizations of reproduction of polynomial spaces (corresponding to $\Lambda=\{(0, \ldots, 0)\}$ ) are given in [7, 9, 21, 23] where, in addition to zero conditions on the symbol $a(\boldsymbol{z})$, extra conditions on $a(\boldsymbol{z})$ and on its derivatives at $(1, \ldots, 1)$ are established.

Characterizations of reproduction properties of binary univariate non-stationary schemes are given in [12] and we extend them here to the case of multivariate non-stationary subdivision with a general integer dilation matrix $M$. We study the so-called non-singular schemes, i.e the ones that generate a zero function if and only if $\mathbf{f}^{[0]}$ is a zero sequence. The main result of our paper states that a nonstationary non-singular subdivision scheme defined by the masks $\left\{\mathbf{a}^{[k]}, k \geq 0\right\}$ reproduces $E P_{\Gamma, \Lambda}$ if and only if there exists $\boldsymbol{\tau} \in \mathbb{R}^{s}$ such that the symbols $a^{[k]}(\boldsymbol{z})$ 
and their derivatives $D^{\gamma} a^{[k]}(\boldsymbol{z})$ satisfy the zero conditions (4.15) and

$$
\mathbf{v}^{\boldsymbol{\gamma}} D^{\boldsymbol{\gamma}} a^{[k]}(\mathbf{v})=|\operatorname{det}(M)| \cdot \mathbf{v}^{M \boldsymbol{\tau}-\boldsymbol{\tau}} \prod_{\ell=1}^{s} \prod_{j=0}^{\gamma_{\ell}-1}\left((M \boldsymbol{\tau}-\boldsymbol{\tau})_{\ell}-j\right),
$$

for all $\mathbf{v}=e^{-\boldsymbol{\lambda} \cdot M^{-(k+1)}}, \gamma \in \Gamma, \boldsymbol{\lambda} \in \Lambda$ and $k \geq 0$, see Theorem 4.7 for details. For a convergent non-stationary subdivision scheme, these conditions are sufficient for reproduction of $E P_{\Gamma, \Lambda}$, see subsection 4.2.1. The parameter $\tau \in \mathbb{R}^{s}$ above is the so-called shift parameter that determines the parametrization

$$
\mathbf{t}_{\boldsymbol{\alpha}}^{[k]}=M^{-k}(\boldsymbol{\alpha}+\boldsymbol{\tau}), \quad \boldsymbol{\alpha} \in \mathbb{Z}^{s}, \quad \boldsymbol{\tau} \in \mathbb{R}^{s}, \quad k \geq 0,
$$

associated to a given non-stationary subdivision scheme. This parametrization specifies to which grid points $\mathbf{t}_{\boldsymbol{\alpha}}^{[k]}$ the newly computed values $f_{\boldsymbol{\alpha}}^{[k]}$ are attached at the $k$-th level of subdivision recursion. The parametrization also influences the starting sequences $\mathbf{f}^{[0]}$. The choice of $\boldsymbol{\tau}$ does not affect either the generation property or convergence of a subdivision scheme, but it affects its reproduction properties. In particular, a correctly chosen shift parameter allows to enrich the variety of shapes the scheme can reproduce.

The paper is organized as follows: in Section 2, we introduce notation and recall some known facts about non-stationary subdivision. In Section 3 for a non-stationary subdivision scheme, we define the notions of generation and reproduction of spaces of exponential polynomials and emphasize the difference between these notions. Since the univariate case is certainly simpler to follow, in Section 4 we first derive the algebraic conditions that characterize the reproduction of exponential polynomials in the univariate $m$-ary case, $M=m$, $m \geq 2$. Then, in Subsection 4.2, we extend this characterization to the multivariate setting. Finally, in Section 5 , we apply the derived algebraic conditions and study subdivision schemes of any arity associated to exponential B-splines and exponential box splines. We also show the effect of the correct choice of $\boldsymbol{\tau}$ and also the effect of the renormalization of subdivision symbols on the reproduction properties of subdivision. We conclude Section 5 with several univariate and bivariate examples. All examples illustrate that our algebraic conditions on subdivision symbols make the construction of new schemes with desired reproduction capabilities and with other enhanced properties more efficient.

\section{Notation and Subdivision Background}

We start this section by setting the notation and continue by recalling some known facts about non-stationary subdivision schemes.

- $\mathbb{N}_{0}$ is the set of natural numbers that includes zero;

- Multi-indices are denoted by Greek boldface letters $\boldsymbol{\alpha}=\left(\alpha_{1}, \ldots, \alpha_{s}\right)^{T} \in$ $\mathbb{N}_{0}^{s}$ 
- Vectors are denoted by boldface letters $\boldsymbol{x} \in \mathbb{R}^{s}$, or $\boldsymbol{z} \in \mathbb{C}^{s}$;

- In the multi-index notation we have $\boldsymbol{x}^{\alpha}=x_{1}^{\alpha_{1}} \cdot \ldots \cdot x_{s}^{\alpha_{s}}, \boldsymbol{\alpha} !=\alpha_{1} ! \ldots \alpha_{s}$ ! and $|\boldsymbol{\alpha}|=\alpha_{1}+\ldots+\alpha_{s}$ for $\boldsymbol{\alpha} \in \mathbb{N}_{0}^{s}$ and $\boldsymbol{x} \in \mathbb{R}^{s}$;

- The product of a real number $y \in \mathbb{R}$ and a vector $\boldsymbol{x}$ is denoted by $y \boldsymbol{x}=$ $\left(y x_{1}, \ldots, y x_{s}\right)^{T}$

- The scalar product of two $s$-dimensional (column) vectors $\boldsymbol{x}$ and $\boldsymbol{z}$ is denoted by $\boldsymbol{x} \cdot \boldsymbol{z}=x_{1} z_{1}+\ldots+x_{s} z_{s}$;

- The scalar product $\boldsymbol{x}^{T} M$ of an $s$-dimensional (column) vector $\boldsymbol{x}$ and a matrix $M$ is denoted by $\boldsymbol{x} \cdot M$;

- For vectors $\boldsymbol{x}$ and $\mathbf{y}$, we have $e^{\boldsymbol{x}}=\left(e^{x_{1}}, \cdots, e^{x_{s}}\right)^{T}$ and $\left(e^{\boldsymbol{x}}\right)^{\mathbf{y}}=e^{\boldsymbol{x} \cdot \mathbf{y}}$;

- Fourier transform $\hat{f}$ of a compactly supported essentially bounded function $f \in L_{\infty}\left(\mathbb{R}^{s}\right)$ is defined by $\hat{f}(\boldsymbol{\omega})=\int_{\mathbb{R}^{s}} f(\boldsymbol{x}) e^{-i \boldsymbol{x} \cdot \boldsymbol{\omega}} d \boldsymbol{x}, \boldsymbol{\omega} \in \mathbb{R}^{s} ;$

- A sequence of real numbers indexed by $\mathbb{Z}^{s}$ is denoted by boldface letters $\mathbf{a}=\left\{\mathrm{a}_{\boldsymbol{\alpha}}, \boldsymbol{\alpha} \in \mathbb{Z}^{s}\right\}$

- A sequence of $s$-dimension vectors indexed by $\mathbb{Z}^{s}$ is denoted by capital boldface letters $\mathbf{T}=\left\{\mathbf{t}_{\boldsymbol{\alpha}}, \boldsymbol{\alpha} \in \mathbb{Z}^{s}\right\}$;

- The space of bounded sequences indexed by $\mathbb{Z}^{s}$ is denoted by $l_{\infty}\left(\mathbb{Z}^{s}\right)$;

- By $D^{\gamma}, \gamma \in \mathbb{N}_{0}^{s}$, we denote a directional derivative.

\subsection{Non-stationary subdivision schemes}

A non-stationary multivariate subdivision scheme is an iterative algorithm with refinement rules

$$
\left(S_{\mathbf{a}^{[k]}} \mathbf{f}^{[k]}\right)_{\boldsymbol{\alpha}}=f_{\boldsymbol{\alpha}}^{[k+1]}=\sum_{\boldsymbol{\beta} \in \mathbb{Z}^{s}} \mathrm{a}_{\boldsymbol{\alpha}-M \boldsymbol{\beta}}^{[k]} f_{\boldsymbol{\beta}}^{[k]}, \quad \boldsymbol{\alpha} \in \mathbb{Z}^{s}, \quad k \geq 0,
$$

and it generates the refined data sequence $\mathbf{f}^{[k+1]}=\left\{f_{\boldsymbol{\alpha}}^{[k+1]}, \boldsymbol{\alpha} \in \mathbb{Z}^{s}\right\}$ from $\mathbf{f}^{[k]}=\left\{f_{\boldsymbol{\alpha}}^{[k]}, \boldsymbol{\alpha} \in \mathbb{Z}^{s}\right\}$. The dilation matrix $M \in \mathbb{Z}^{s \times s}$ is assumed to have all its eigenvalues greater than 1 in absolute value. Subdivision schemes are based on the application of the subdivision operators $S_{\mathbf{a}^{[k]}}$ constructed from the socalled subdivision mask $\mathbf{a}^{[k]}=\left\{\mathrm{a}_{\boldsymbol{\alpha}}^{[k]}, \boldsymbol{\alpha} \in \mathbb{Z}^{s}\right\}$ at level $k$. Each subdivision mask is assumed to be of finite support, i.e. $\operatorname{supp}\left(\mathbf{a}^{[k]}\right) \subset\left[0, N_{k}\right]^{s}$ for some positive integer $N_{k}$. The (non-stationary) subdivision scheme is denoted by $S_{\left\{\mathbf{a}^{[k]}, k \geq 0\right\}}$ and is given by

$$
\left\{\begin{array}{l}
\text { Input: } \quad \mathbf{f}^{[0]}, \quad\left\{\mathbf{a}^{[k]}, k \geq 0\right\} \\
\text { For } k=0,1,2, \ldots, \\
\quad \mathbf{f}^{[k+1]}=S_{\mathbf{a}^{[k]}} \mathbf{f}^{[k]}
\end{array}\right.
$$


for some initial data $\mathbf{f}^{[0]}=\left\{f_{\boldsymbol{\alpha}}^{[0]}, \boldsymbol{\alpha} \in \mathbb{Z}^{s}\right\}$.

The symbols of a non-stationary subdivision scheme are given by Laurent polynomials

$$
a^{[k]}(\boldsymbol{z})=\sum_{\boldsymbol{\alpha} \in \mathbb{Z}^{s}} \mathrm{a}_{\boldsymbol{\alpha}}^{[k]} \boldsymbol{z}^{\boldsymbol{\alpha}}, \quad \boldsymbol{z} \in(\mathbb{C} \backslash\{0\})^{s}, \quad k \geq 0 .
$$

Denote $m=|\operatorname{det}(M)|$ and by $E$ the set of representatives of $\mathbb{Z}^{s} / M \mathbb{Z}^{s}$. Clearly, $E$ contains $\mathbf{0}=(0, \ldots, 0)^{T}$. Define the set

$$
\Xi=\left\{e^{2 \pi i M^{-T} \boldsymbol{\xi}}: \boldsymbol{\xi} \text { is a coset representative of } \mathbb{Z}^{s} / M^{T} \mathbb{Z}^{s}\right\},
$$

which contains $\mathbf{1}=(1,1, \ldots, 1)^{T}$. The $m$ sub-masks of the masks $\mathbf{a}^{[k]}$ are

$$
\left\{\mathrm{a}_{\boldsymbol{\varepsilon}+M \boldsymbol{\alpha}}^{[k]}, \boldsymbol{\alpha} \in \mathbb{Z}^{s}\right\}, \quad \varepsilon \in E, \quad k \geq 0,
$$

and their symbols (sub-symbols of the masks) are

$$
a_{\boldsymbol{\varepsilon}}^{[k]}(\boldsymbol{z})=\sum_{\boldsymbol{\alpha} \in \mathbb{Z}^{s}} \mathrm{a}_{\boldsymbol{\varepsilon}+M \boldsymbol{\alpha}}^{[k]} \boldsymbol{z}^{\boldsymbol{\varepsilon}+M \boldsymbol{\alpha}}, \quad \boldsymbol{\varepsilon} \in E, \quad k \geq 0,
$$

respectively. The symbols satisfy

$$
a^{[k]}(\boldsymbol{z})=\sum_{\boldsymbol{\varepsilon} \in E} a_{\boldsymbol{\varepsilon}}^{[k]}(\boldsymbol{z}), \quad k \geq 0 .
$$

For a given parametrization $\mathbf{T}^{[k]}=\left\{\mathbf{t}_{\boldsymbol{\alpha}}^{[k]}, \boldsymbol{\alpha} \in \mathbb{Z}^{s}\right\}$ in (1.2), a notion of convergence for $S_{\left\{\mathbf{a}^{[k]}, k \geq 0\right\}}$ is established using the sequence $\left\{F^{[k]}, k \geq 0\right\}$ of continuous functions $F^{[k]}$ that interpolate the data $\mathbf{f}^{[k]}$ at the parameter values $\mathbf{t}_{\boldsymbol{\alpha}}^{[k]}, \boldsymbol{\alpha} \in \mathbb{Z}^{s}$, namely

$$
F^{[k]}\left(\mathbf{t}_{\boldsymbol{\alpha}}^{[k]}\right)=f_{\boldsymbol{\alpha}}^{[k]}, \quad \boldsymbol{\alpha} \in \mathbb{Z}^{s}, \quad k \geq 0 .
$$

The scheme $S_{\left\{\mathbf{a}^{[k]}, k \geq 0\right\}}$ applied to initial data $\mathbf{f}^{[0]}$ is called convergent, if there exists a continuous limit function $g_{\mathbf{f}^{[0]}}$ (which is nonzero for at least one initial nonzero sequence) such that the sequence $\left\{F^{[k]}, k \geq 0\right\}$ converges uniformly to $g_{\mathbf{f}^{[0]}}$, i.e.

$$
g_{\mathbf{f}^{[0]}}=\lim _{k \rightarrow \infty} S_{\mathbf{a}^{[k]}} S_{\mathbf{a}^{[k-1]}} \cdots S_{\mathbf{a}^{[0]}} \mathbf{f}^{[0]}=\lim _{k \rightarrow \infty} F^{[k]} .
$$

The scheme $S_{\left\{\mathbf{a}^{[k]}, k \geq 0\right\}}$ is called weakly convergent, if the sequence $\left\{F^{[k]}, k \geq 0\right\}$ in (2.7) converges pointwise to $g_{\mathbf{f}^{[0]}} \in L_{\infty}\left(\mathbb{R}^{s}\right)$.

Due to linearity of each subdivision operator $S_{\mathbf{a}^{[k]}}$, the limit functions $g_{\mathbf{f}^{[0]}}$ exist if and only if the subdivision scheme applied to the initial data $\boldsymbol{\delta}=\left\{\delta_{\boldsymbol{\alpha}, \mathbf{0}}, \boldsymbol{\alpha} \in \mathbb{Z}^{s}\right\}$ converges to the so-called basic limit function $\phi=g_{\delta}$. In this case,

$$
g_{\mathbf{f}^{[0]}}=\sum_{\boldsymbol{\alpha} \in \mathbb{Z}^{s}} f_{\boldsymbol{\alpha}}^{[0]} \phi(\cdot-\boldsymbol{\alpha}) .
$$

Differently from the stationary case where all masks $\mathbf{a}^{[k]}$ are the same, in the non-stationary setting, one could start the subdivision process with a mask 
at level $\ell \geq 0$ and get a family of subdivision schemes based on the masks $\left\{\mathbf{a}^{[\ell+k]}, k \geq 0\right\}, \ell \geq 0$. The corresponding subdivision limits are denoted by

$$
g_{\mathbf{f}}^{[\ell]}:=\lim _{k \rightarrow \infty} S_{\mathbf{a}^{[\ell+k]}} S_{\mathbf{a}^{[\ell+k-1]}} \cdots S_{\mathbf{a}^{[\ell]}} \mathbf{f}^{[0]}, \quad \ell \geq 0 .
$$

An interesting fact about the compactly supported basic limit functions $\phi^{[\ell]}:=$ $g_{\delta}^{[\ell]}, \ell \geq 0,\left(\phi^{[0]}=\phi\right)$ is that they are mutually refinable, i.e., they satisfy the functional equations

$$
\phi^{[\ell]}=\sum_{\boldsymbol{\alpha} \in \mathbb{Z}^{s}} \mathrm{a}_{\boldsymbol{\alpha}}^{[\ell]} \phi^{[\ell+1]}(M \cdot-\boldsymbol{\alpha}), \quad \ell \geq 0, \quad \text { with } \mathbf{a}^{[\ell]} \text { the } \ell \text {-th level mask. }
$$

In this paper, we consider subdivision schemes that are non-singular, i.e. they are convergent (or weakly convergent) and, such that $g_{\mathbf{f}^{[0]}}=0$ if and only if $\mathbf{f}^{[0]}=0$. The following proposition connects the non-singularity of a nonstationary scheme with the linear independence of the translates of $\phi^{[\ell]}, \ell \geq 0$.

Proposition 2.1. If a subdivision scheme $S_{\left\{\mathbf{a}^{[k]}, k \geq 0\right\}}$ is non-singular, then the integer translates of $\phi^{[\ell]}$ are linearly independent for each $\ell \geq 0$.

Proof: If the convergent subdivision scheme $S_{\left\{\mathbf{a}^{[k]}, k \geq 0\right\}}$ is non-singular, then for any starting sequence $\mathbf{d}=\left\{\mathrm{d}_{\boldsymbol{\alpha}}, \boldsymbol{\alpha} \in \mathbb{Z}^{s}\right\}$ we have

$$
\lim _{k \rightarrow \infty} S_{\mathbf{a}^{[k+\ell]}} \ldots S_{\mathbf{a}^{[\ell]}} \mathbf{d}=\sum_{\boldsymbol{\alpha} \in \mathbb{Z}^{s}} \mathrm{~d}_{\boldsymbol{\alpha}} \phi^{[\ell]}(\cdot-\boldsymbol{\alpha})=0
$$

if and only if $\mathbf{d}$ is the zero sequence.

We formulate the converse of Proposition 2.1 separately as it requires additional assumptions on the functions $\phi^{[\ell]}$.

Proposition 2.2. Let $\phi^{[\ell]} \in L_{\infty}\left(\mathbb{R}^{s}\right), \ell \geq 0$, be compactly supported solutions of the refinement equations (2.9) and such that their integer shifts are linearly independent for each $\ell$. If there exists $L \geq 0$ such that $\phi^{[\ell]}$ additionally satisfy

$$
C\|\mathbf{f}\|_{\infty} \leq\left\|\sum_{\boldsymbol{\alpha} \in \mathbb{Z}^{s}} f_{\boldsymbol{\alpha}} \phi^{[\ell]}(\cdot-\boldsymbol{\alpha})\right\|_{\infty}, \quad \mathbf{f} \in l_{\infty}\left(\mathbb{Z}^{s}\right), \quad \ell \geq L,
$$

then the scheme $S_{\left\{\mathbf{a}^{[k]}, k \geq 0\right\}}$ is non-singular.

Proof: By [15, Theorem 13], the assumptions on $\phi^{[\ell]}$ guarantee that the associated subdivision scheme $S_{\left\{\mathbf{a}^{[k]}, k \geq 0\right\}}$ is convergent. The linear independence of $\phi^{[\ell]}$ yields the claim. 


\section{Exponential polynomials and non-stationary subdivision schemes}

In this section, for a non-stationary subdivision scheme, we define the notions of exponential polynomial generation and exponential polynomial reproduction. We start by defining the space of exponential polynomials on $\mathbb{R}^{s}$.

Definition 3.1. For $\Gamma \subset \mathbb{N}_{0}^{s}$ and $\Lambda \subset \mathbb{C}^{s}$ we define

$$
E P_{\Gamma, \Lambda}=\operatorname{span}\left\{\boldsymbol{x}^{\boldsymbol{\gamma}} e^{\boldsymbol{\lambda} \cdot \boldsymbol{x}}, \quad \gamma \in \Gamma, \boldsymbol{\lambda} \in \Lambda\right\} .
$$

The following two observations motivate our interest in the function space $E P_{\Gamma, \Lambda}$.

Remark 3.2. (i) An exponential polynomial $p(\boldsymbol{x})=\boldsymbol{x}^{\gamma} e^{\boldsymbol{\lambda} \boldsymbol{x}} \in E P_{\Gamma, \Lambda}$ is a polynomial, if $\boldsymbol{\lambda}=\mathbf{0}$, or is an exponential function, if $\boldsymbol{\gamma}=\mathbf{0}$. If $\boldsymbol{\gamma}=\mathbf{0}$ and $\boldsymbol{\lambda} \in i \mathbb{R}^{s}$, then $p$ is a trigonometric function, or a hyperbolic function, if $\boldsymbol{\gamma}=\mathbf{0}$ and $\boldsymbol{\lambda} \in \mathbb{R}^{s}$. The reproduction and generation of $E_{\Gamma, \Lambda}$ or its subspaces by non-stationary subdivision are important in CAGD, motion planning or isogeometric analysis.

(ii) We would like to emphasize that the definitions and the proofs of the results of this Section and Section 4 still apply if one works with a subspace $E P_{Q}$ of $E P_{\Gamma, \Lambda}$, where $Q \subset \Gamma \times \Lambda$ consists of pairs $(\boldsymbol{\gamma}, \boldsymbol{\lambda})$ for some $\boldsymbol{\gamma} \in \Gamma$ and $\lambda \in \Lambda$. In this case, the algebraic conditions in (4.2), (4.13), (4.15), (4.21) and (4.23) should be checked for corresponding pairs

$$
(\mathbf{v}, \gamma), \quad \mathbf{v}=\left(\epsilon_{1} e^{-\left(\boldsymbol{\lambda} \cdot M^{-(k+1)}\right)_{1}}, \ldots, \epsilon_{s} e^{-\left(\boldsymbol{\lambda} \cdot M^{-(k+1)}\right)_{s}}\right), \quad \boldsymbol{\epsilon} \in \Xi, \quad(\boldsymbol{\lambda}, \gamma) \in Q
$$

Since most of the properties of a subdivision scheme, e.g. its convergence, smoothness or its support size, do not depend on the choice of $\mathbf{T}^{[k]}=\left\{\mathbf{t}_{\boldsymbol{\alpha}}^{[k]}, \boldsymbol{\alpha} \in\right.$ $\left.\mathbb{Z}^{s}\right\}$, these are usually set to

$$
\mathbf{t}_{\boldsymbol{\alpha}}^{[k]}=M^{-k} \boldsymbol{\alpha}, \quad \boldsymbol{\alpha} \in \mathbb{Z}^{s}, \quad k \geq 0 .
$$

We refer to the choice in (3.1) as standard parametrization. On the contrary, the capability of subdivision to reproduce exponential polynomials does depend on the choice of $\mathbf{t}_{\boldsymbol{\alpha}}^{[k]}$ and the standard parametrization is not always the optimal one. We show in this section that the choice

$$
\mathbf{t}_{\boldsymbol{\alpha}}^{[k]}=M^{-k}(\boldsymbol{\alpha}+\boldsymbol{\tau}), \quad \boldsymbol{\alpha} \in \mathbb{Z}^{s}, \quad k \geq 0,
$$

with a suitable $\tau \in \mathbb{R}^{s}$ turns out to be more advantageous.

We call the sequence $\left\{\mathbf{T}^{[k]}, k \geq 0\right\}$ with $\mathbf{T}^{[k]}=\left\{M^{-k}(\boldsymbol{\alpha}+\boldsymbol{\tau}), \boldsymbol{\alpha} \in \mathbb{Z}^{s}\right\}$ the parametrization associated with a subdivision scheme and $\boldsymbol{\tau} \in \mathbb{R}^{s}$ the corresponding shift parameter.

Definition 3.3. A convergent subdivision scheme $S_{\left\{\mathbf{a}^{[k]}, k \geq 0\right\}}$ is said to be $E P_{\Gamma, \Lambda}$-generating, if there exists a parametrization $\left\{\mathbf{T}^{[k]}, k \geq 0\right\}$ with $\mathbf{T}^{[k]}=$ 
$\left\{\mathbf{t}_{\boldsymbol{\alpha}}^{[k]}=M^{-k}(\boldsymbol{\alpha}+\boldsymbol{\tau}), \boldsymbol{\alpha} \in \mathbb{Z}^{s}\right\}$ and $\boldsymbol{\tau} \in \mathbb{R}^{s}$ such that for every initial sequence $\mathbf{f}^{[0]}=\left\{p\left(\mathbf{t}_{\boldsymbol{\alpha}}^{[0]}\right), \boldsymbol{\alpha} \in \mathbb{Z}^{s}\right\}, p \in E P_{\Gamma, \Lambda}$, we have

$$
\lim _{k \rightarrow \infty} S_{\mathbf{a}^{[\ell+k]}} S_{\mathbf{a}^{[\ell+k-1]}} \ldots S_{\mathbf{a}^{[\ell]}} \mathbf{f}^{[0]} \in E P_{\Gamma, \Lambda} \quad \forall \ell \geq 0 .
$$

We continue by defining the notion of $E P_{\Gamma, \Lambda}$-reproduction.

Definition 3.4. A convergent subdivision scheme $S_{\left\{\mathbf{a}^{[k]}, k \geq 0\right\}}$ is said to be $E P_{\Gamma, \Lambda}$-reproducing, if there exists a parametrization $\left\{\mathbf{T}^{[k]}, k \geq 0\right\}$ with $\mathbf{T}^{[k]}=$ $\left\{\mathbf{t}_{\boldsymbol{\alpha}}^{[k]}=M^{-k}(\boldsymbol{\alpha}+\boldsymbol{\tau}), \boldsymbol{\alpha} \in \mathbb{Z}^{s}\right\}$ and $\boldsymbol{\tau} \in \mathbb{R}^{s}$ such that for every initial sequence $\mathbf{f}^{[0]}=\left\{p\left(\mathbf{t}_{\boldsymbol{\alpha}}^{[0]}\right), \boldsymbol{\alpha} \in \mathbb{Z}^{s}\right\}, p \in E P_{\Gamma, \Lambda}$, we have

$$
\lim _{k \rightarrow \infty} S_{\mathbf{a}^{[\ell+k]}} S_{\mathbf{a}^{[\ell+k-1]}} \cdots S_{\mathbf{a}^{[\ell]}} \mathbf{f}^{[0]}=p \quad \forall \ell \geq 0 .
$$

Note that the generation and the reproduction properties are independent of the starting level $\ell$ of refinement.

We define next the step-wise reproduction property of subdivision which is easier to check than its $E P_{\Gamma, \Lambda}$-reproduction.

Definition 3.5. A convergent subdivision scheme $S_{\left\{\mathbf{a}^{[k]}, k \geq 0\right\}}$ is said to be stepwise $E P_{\Gamma, \Lambda}$-reproducing, if there exists a parametrization $\left\{\mathbf{T}^{[k]}, k \geq 0\right\}$ with $\mathbf{T}^{[k]}=\left\{\mathbf{t}_{\boldsymbol{\alpha}}^{[k]}=M^{-k}(\boldsymbol{\alpha}+\boldsymbol{\tau}), \boldsymbol{\alpha} \in \mathbb{Z}^{s}\right\}$ and $\boldsymbol{\tau} \in \mathbb{R}^{s}$ such that for the sequences $\mathbf{f}^{[k]}=\left\{p\left(\mathbf{t}_{\boldsymbol{\alpha}}^{[k]}\right), \boldsymbol{\alpha} \in \mathbb{Z}^{s}\right\}, k \geq 0$, and $p \in E P_{\Gamma, \Lambda}$, we have

$$
\mathbf{f}^{[k+1]}=S_{\mathbf{a}^{[k]}} \mathbf{f}^{[k]} .
$$

It has been already observed in [7] in the stationary multivariate case and in [12] in the binary non-stationary univariate setting that for non-singular schemes the concepts of reproduction and step-wise reproduction are equivalent. Since these results extend easily to the multivariate case we state the following proposition without a proof.

Proposition 3.6. A non-singular non-stationary subdivision scheme is stepwise $E P_{\Gamma, \Lambda}$-reproducing if and only if it is $E P_{\Gamma, \Lambda}$-reproducing.

\section{Algebraic conditions for generation and reproduction of exponen- tial polynomials}

In this section we derive algebraic conditions that guarantee $E P_{\Gamma, \Lambda}$-reproduction by non-stationary subdivision schemes. These conditions are given in terms of the symbols $\left\{a^{[k]}, k \geq 0\right\}$ which are evaluated at the elements of the sets

$$
V_{k}=\left\{\left(v_{1}, \ldots, v_{s}\right)^{T}: v_{j}=\epsilon_{j} e^{-\left(\boldsymbol{\lambda} \cdot M^{-(k+1)}\right)_{j}}, \boldsymbol{\lambda} \in \Lambda, \boldsymbol{\epsilon} \in \Xi\right\}, \quad k \geq 0 .
$$

For $k \geq 0$, we also define the following sets of vectors

$$
V_{k}^{\prime}=\left\{\left(v_{1}, \ldots, v_{s}\right)^{T}: v_{j}=\epsilon_{j} e^{-\left(\boldsymbol{\lambda} \cdot M^{-(k+1)}\right)_{j}}, \boldsymbol{\lambda} \in \Lambda, \boldsymbol{\epsilon} \in \Xi \backslash\{\mathbf{1}\}\right\} .
$$

To simplify the presentation of the results presented in this section, in subsection 4.1, we consider the univariate case first and then extend our results to the multivariate setting in subsection 4.2 . 


\subsection{Univariate case}

In the univariate case, $M=m \geq 2, E=\{0, \ldots, m-1\}$ and $\Xi=\left\{e^{2 \pi i m^{-1} \varepsilon}\right.$ : $\varepsilon \in E\}$ consists of the $m$-th roots of unity. From (2.1), we get that the $m$-ary subdivision scheme is given by the repeated application of $m$ different rules

$$
\left(S_{\mathbf{a}^{[k]}} \mathbf{f}^{[k]}\right)_{m \alpha+\varepsilon}=f_{m \alpha+\varepsilon}^{[k+1]}=\sum_{\beta \in \mathbb{Z}} \mathrm{a}_{m \beta+\varepsilon}^{[k]} f_{\alpha-\beta}^{[k]}, \quad \alpha \in \mathbb{Z}, \quad \varepsilon \in E, \quad k \geq 0 .
$$

The structure of the sets $V_{k}, V_{k}^{\prime}, k \geq 0$, is a lot simpler in the univariate case, namely,

$$
V_{k}=\left\{\epsilon e^{-\lambda m^{-(k+1)}}: \lambda \in \Lambda, \epsilon \in \Xi\right\},
$$

and

$$
V_{k}^{\prime}=\left\{\epsilon e^{-\lambda m^{-(k+1)}}: \lambda \in \Lambda, \epsilon \in \Xi \backslash\{1\}\right\},
$$

for $k \geq 0$, respectively.

Remark 4.1. We remark that since our goal is to make the multivariate extension of univariate results as straightforward as possible, in this subsection we use notation less common for the univariate setting. For example, we write $\sum_{\varepsilon \in E} \mathrm{c}_{\varepsilon}, E=\{0, \ldots, m-1\}$ instead of $\sum_{j=0}^{m-1} \mathrm{c}_{j}$.

\subsubsection{Generation of exponential polynomials}

The following result characterizes the $E P_{\Gamma, \Lambda}$-generation of a non-singular scheme in terms of the so-called zero conditions (4.2). The proof of Proposition 4.2 in the case $m=2$ is given in [36, Theorem 1]. We give the generalization of this result to the case $m \geq 2$ in the notation familiar to subdivision audience.

Proposition 4.2. A non-singular non-stationary subdivision scheme defined by the symbols $\left\{a^{[k]}(z), k \geq 0\right\}$ is $E P_{\Gamma, \Lambda}$-generating if and only if

$$
D^{\gamma} a^{[k]}(v)=0, \quad \gamma \in \Gamma, \quad v \in V_{k}^{\prime}, \quad k \geq 0,
$$

for $V_{k}^{\prime}=\left\{\epsilon e^{-\lambda m^{-(k+1)}}: \lambda \in \Lambda, \epsilon \in \Xi \backslash\{1\}\right\}$.

Proof. Let $\ell \geq 0$ and $\lambda \in \Lambda$. We multiply both sides of the non-stationary refinement equation (2.9) by $e^{-\lambda m^{-\ell} x}, x \in \mathbb{R}$, and get

$$
e^{-\lambda m^{-\ell} x} \phi^{[\ell]}(x)=\sum_{\alpha \in \mathbb{Z}} \mathrm{a}_{\alpha}^{[\ell]} e^{-\lambda m^{-(\ell+1)} \alpha} \phi^{[\ell+1]}(m x-\alpha) e^{-\lambda m^{-(\ell+1)}(m x-\alpha)} .
$$

Set $\Phi_{\ell}(x)=e^{-\lambda m^{-\ell} x} \phi^{[\ell]}(x)$. Then, equation (4.3) becomes

$$
\Phi_{\ell}(x)=\sum_{\alpha \in \mathbb{Z}} \mathrm{a}_{\alpha}^{[\ell]} e^{-\lambda m^{-(\ell+1)} \alpha} \Phi_{\ell+1}(m x-\alpha),
$$


or, equivalently, on the Fourier side,

$$
\widehat{\Phi}_{\ell}(\omega)=m^{-1} a^{[\ell]}\left(e^{-\lambda m^{-(\ell+1)}-i m^{-1} \omega}\right) \widehat{\Phi}_{\ell+1}\left(m^{-1} \omega\right),
$$

where $a^{[\ell]}\left(e^{-i \omega}\right)=\sum_{\alpha \in \mathbb{Z}} \mathrm{a}_{\alpha}^{[\ell]} e^{-i \omega \alpha}, \quad \omega \in \mathbb{R}$. The integer shifts of $\phi^{[\ell]}$ are $E P_{\Gamma, \Lambda^{-}}$ generating, i.e.

$$
E P_{\Gamma, \Lambda} \subset \operatorname{span}\left\{\phi^{[\ell]}(x-\alpha): \alpha \in \mathbb{Z}\right\},
$$

if and only if the functions $\Phi_{\ell}$ for all $\lambda \in \Lambda$ satisfy the so-called Strang-Fix conditions

$$
\left.D^{\gamma} \widehat{\Phi}_{\ell}(\omega)\right|_{\omega=2 \pi \beta}=0 \quad \text { for } \quad \beta \in E \backslash\{0\}, \quad \gamma \in \Gamma,
$$

and $\widehat{\Phi}_{\ell}(0) \neq 0$, see e.g. 35 .

Therefore, evaluation of (4.5) at $2 \pi \beta, \beta \in E \backslash\{0\}$, yields

$$
0=\widehat{\Phi}_{\ell}(2 \pi \beta)=m^{-1} a^{[\ell]}\left(e^{-\lambda m^{-(\ell+1)}-2 i \pi m^{-1} \beta}\right) \widehat{\Phi}_{\ell+1}\left(2 \pi m^{-1} \beta\right) .
$$

By Proposition 2.1, the system of functions $\left\{\Phi_{\ell}(\cdot-\alpha): \alpha \in \mathbb{Z}\right\}$ is linearly independent for each $\ell \geq 0$. By [32], its linear independence is equivalent to the fact that the set $\left\{\xi \in \mathbb{C}: \widehat{\Phi}_{\ell}(\xi+2 \pi \beta)=0\right.$ for all $\left.\beta \in \mathbb{Z}\right\}$ is empty. Therefore, with $\xi=2 \pi m^{-1} \beta \in \mathbb{C}$, the identities (4.6) are satisfied if and only if

$$
a^{[\ell]}(v)=0, \quad v \in V_{\ell}^{\prime}, \quad \ell \geq 0 .
$$

Taking derivatives of both sides of (4.5)

$$
D^{\gamma} \widehat{\Phi_{\ell}}(\omega)=m^{-1} \sum_{\eta \leq \gamma}\left(\begin{array}{c}
\gamma \\
\eta
\end{array}\right) D^{\eta} a^{[\ell]}\left(e^{-\lambda m^{-(\ell+1)}-i m^{-1} \omega}\right) D^{\gamma-\eta} \widehat{\Phi}_{\ell+1}\left(m^{-1} \omega\right)
$$

and evaluating them at $2 \pi \beta, \beta \in E \backslash\{0\}$ yields, by induction on $\gamma$, that the non-singular scheme is $E P_{\Gamma, \Lambda}$-generating if and only if the identities in (4.2) are satisfied.

We would like to emphasize that the generation properties of subdivision schemes are well-understood. Our interest lies in better understanding of their reproduction properties.

\subsubsection{Reproduction of exponential polynomials}

In this subsection, in Theorem 4.4, we derive algebraic conditions on the mask symbols $\left\{a^{[k]}(z), k \geq 0\right\}$ that characterize the $E P_{\Gamma, \Lambda}$-reproduction property of the associated non-stationary subdivision scheme. We start by proving Proposition 4.3 that constitutes the main part of the proof of Theorem 4.4.

Proposition 4.3. A subdivision scheme $S_{\left\{\mathbf{a}^{[k]}, k \geq 0\right\}}$ is step-wise $E P_{\Gamma, \Lambda}$-reproducing if and only if there exists a shift parameter $\tau \in \mathbb{R}$ such that

$$
\sum_{\beta \in \mathbb{Z}} \mathrm{a}_{m \beta+\varepsilon}^{[k]}(m \beta)^{\gamma^{\prime}} v^{m \beta+\varepsilon}=v^{m \tau-\tau} \epsilon^{-m \tau+\tau+\varepsilon}(m \tau-\tau-\varepsilon)^{\gamma^{\prime}}
$$


is satisfied for all $v \in V_{k}=\left\{\epsilon e^{-\lambda m^{-(k+1)}}: \lambda \in \Lambda, \epsilon \in \Xi\right\}, k \geq 0, \gamma^{\prime} \leq \gamma$, $\gamma \in \Gamma, \varepsilon \in E$ and $\epsilon \in \Xi$.

Proof: Let $\gamma \in \Gamma, \lambda \in \Lambda$ and define

$$
f_{\alpha}^{[k]}=\left(m^{-k}(\alpha+\tau)\right)^{\gamma} \cdot e^{\lambda m^{-k}(\alpha+\tau)}, \quad \alpha \in \mathbb{Z}, \quad k \geq 0 .
$$

By (4.1) and by definition 3.5, the step-wise reproduction of this sequence is equivalent to the existence of $\tau \in \mathbb{R}$ such that

$$
f_{m \alpha+\varepsilon}^{[k+1]}=\sum_{\beta \in \mathbb{Z}} \mathrm{a}_{m \beta+\varepsilon}^{[k]} f_{\alpha-\beta}^{[k]}, \quad \varepsilon \in E, \quad \alpha \in \mathbb{Z}, \quad k \geq 0 .
$$

Multiplying both sides of (4.7) by $e^{-\lambda m^{-(k+1)}(m \alpha+m \tau+\varepsilon)}$ we get

$$
\begin{aligned}
\sum_{\beta \in \mathbb{Z}} \mathrm{a}_{m \beta+\varepsilon}^{[k]}\left(m^{-k}(\alpha-\beta+\tau)\right)^{\gamma} e^{-\lambda m^{-(k+1)}(m \beta+\varepsilon)} \\
=\left(m^{-(k+1)}(m \alpha+\varepsilon+\tau)\right)^{\gamma} e^{-\lambda m^{-(k+1)}(m \tau-\tau)},
\end{aligned}
$$

or, equivalently,

$$
\begin{aligned}
\sum_{\beta \in \mathbb{Z}} \mathrm{a}_{m \beta+\varepsilon}^{[k]}\left(m^{-k}(\alpha-\beta+\tau)\right)^{\gamma} e^{\left(-\lambda m^{-(k+1)}-2 \pi i m^{-1} \varepsilon\right) \cdot(m \beta+\varepsilon)} & \\
= & e^{-2 \pi i m^{-1} \varepsilon \cdot(m \beta+\varepsilon-m \tau+\tau)}\left(m^{-(k+1)}(m \alpha+\varepsilon+\tau)\right)^{\gamma} e^{\left(-\lambda m^{-(k+1)}-2 \pi i m^{-1} \varepsilon\right)(m \tau-\tau)} .
\end{aligned}
$$

Due to the fact that

$$
e^{2 \pi i m^{-1} \varepsilon \cdot m \beta}=1 \text { for all } \varepsilon \in E \text { and } \beta \in \mathbb{Z},
$$

we have that (4.8) is equivalent to

$$
\begin{aligned}
\sum_{\beta \in \mathbb{Z}} \mathrm{a}_{m \beta+\varepsilon}^{[k]}\left(m^{-k}(\alpha-\beta+\tau)\right)^{\gamma} v^{m \beta+\varepsilon} & \\
= & \epsilon^{-m \tau+\tau+\varepsilon}\left(m^{-(k+1)}(m \alpha+\varepsilon+\tau)\right)^{\gamma} v^{m \tau-\tau}
\end{aligned}
$$

for $v \in V_{k}$. Considering both sides of (4.9) as polynomials in $m^{-k} \alpha$ with

$$
\left(m^{-k}(\alpha-\beta+\tau)\right)^{\gamma}=\sum_{\eta \leq \gamma}\left(\begin{array}{c}
\gamma \\
\eta
\end{array}\right)\left(m^{-k}(-\beta+\tau)\right)^{\eta}\left(m^{-k} \alpha\right)^{\gamma-\eta},
$$

and

$$
\left(m^{-(k+1)}(m \alpha+\varepsilon+\tau)\right)^{\gamma}=\sum_{\eta \leq \gamma}\left(\begin{array}{l}
\gamma \\
\eta
\end{array}\right)\left(m^{-(k+1)}(\varepsilon+\tau)\right)^{\eta}\left(m^{-k} \alpha\right)^{\gamma-\eta},
$$


we get that (4.9) is equivalent to

$$
\begin{aligned}
& \sum_{\beta \in \mathbb{Z}} \mathrm{a}_{m \beta+\varepsilon}^{[k]}\left(m^{-k}(-\beta+\tau)\right)^{\eta} v^{m \beta+\varepsilon} \\
& \quad=\epsilon^{-m \tau+\tau+\varepsilon}\left(m^{-(k+1)}(\varepsilon+\tau)\right)^{\eta} v^{m \tau-\tau}, \quad v \in V_{k}, \quad \eta \leq \gamma
\end{aligned}
$$

Next, we expand

$$
\left(m^{-(k+1)}(m \tau+\tau-m \tau+\varepsilon)\right)^{\eta}=\sum_{\gamma^{\prime} \leq \eta}\left(\begin{array}{c}
\eta \\
\gamma^{\prime}
\end{array}\right)\left(m^{-(k+1)}(\tau-m \tau+\varepsilon)\right)^{\gamma^{\prime}}\left(m^{-k} \tau\right)^{\eta-\gamma^{\prime}}
$$

and, similarly, $\left(m^{-k}(-\beta+\tau)\right)^{\eta}$. Thus, 4.10) is satisfied if and only if

$$
\begin{aligned}
& \sum_{\beta \in \mathbb{Z}} \mathrm{a}_{m \beta+\varepsilon}^{[k]}\left(-m^{-k} \beta\right)^{\gamma^{\prime}} v^{m \beta+\varepsilon}= \\
& \epsilon^{-m \tau+\tau+\varepsilon}\left(m^{-(k+1)}(\tau+\varepsilon-m \tau)\right)^{\gamma^{\prime}} v^{m \tau-\tau}, v \in V_{k}, \gamma^{\prime} \leq \eta \leq \gamma
\end{aligned}
$$

Multiplying both sides by $\left(-m^{k+1}\right)^{\gamma^{\prime}}$, we get the claim.

To formulate the main result of this subsection we define

$$
q_{0}(z)=1, \quad q_{\gamma}(z)=\prod_{j=0}^{\gamma-1}(z-j), \quad \gamma \in \mathbb{N}_{0}, \quad z \in \mathbb{C} .
$$

Theorem 4.4. A non-singular subdivision scheme $S_{\left\{\mathbf{a}^{[k]}, k \geq 0\right\}}$ reproduces $E P_{\Gamma, \Lambda}$ if and only if there exists a shift parameter $\tau \in \mathbb{R}$ such that

$$
v^{\gamma} D^{\gamma} a^{[k]}(v)= \begin{cases}m \cdot v^{m \tau-\tau} q_{\gamma}(m \tau-\tau), & \text { for all } v \text { such that } \epsilon=1, \\ 0, & \text { otherwise }\end{cases}
$$

for all $v \in V_{k}=\left\{\epsilon e^{-\lambda m^{-(k+1)}}: \lambda \in \Lambda, \epsilon \in \Xi\right\}, k \geq 0, \gamma \in \Gamma$.

Proof: Due to Proposition [3.6 it suffices to prove this statement for stepwise $E P_{\Gamma, \Lambda}$-reproduction. We use Proposition 4.3 and show that the step-wise $E P_{\Gamma, \Lambda}$-reproduction, is equivalent to the identities (4.13). To this purpose we consider $q_{\gamma}(m \alpha+\varepsilon)$, for fixed $\gamma \in \Gamma$ and $\varepsilon \in E$, as a polynomial in $m \alpha$ and write

$$
q_{\gamma}(m \alpha+\varepsilon)=\sum_{\eta \leq \gamma} c_{\gamma, \varepsilon, \eta}(m \alpha)^{\eta}, \quad c_{\gamma, \varepsilon, \eta} \in \mathbb{R} .
$$

From (2.6) we get

$$
z^{\gamma} D^{\gamma} a^{[k]}(z)=\sum_{\varepsilon \in E} \sum_{\alpha \in \mathbb{Z}} \mathrm{a}_{m \alpha+\varepsilon}^{[k]} q_{\gamma}(m \alpha+\varepsilon) z^{m \alpha+\varepsilon}, \quad \gamma \in \Gamma .
$$


Let $v \in V_{k}$. By Proposition 4.3 and by (4.14), the step-wise $E P_{\Gamma, \Lambda}$-reproduction is equivalent to

$$
\begin{aligned}
v^{\gamma} D^{\gamma} a^{[k]}(v) & =\sum_{\varepsilon \in E} \sum_{\alpha \in \mathbb{Z}} \mathrm{a}_{m \alpha+\varepsilon}^{[k]} q_{\gamma}(m \alpha+\varepsilon) v^{m \alpha+\varepsilon} \\
& =\sum_{\varepsilon \in E} \sum_{\eta \leq \gamma} c_{\gamma, \varepsilon, \eta} \sum_{\alpha \in \mathbb{Z}} \mathrm{a}_{m \alpha+\varepsilon}^{[k]}(m \alpha)^{\eta} v^{m \alpha+\varepsilon} \\
& =v^{m \tau-\tau} \sum_{\varepsilon \in E} \epsilon^{-m \tau+\tau+\varepsilon} \sum_{\eta \leq \gamma} c_{\gamma, \varepsilon, \eta}(m \tau-\tau-\varepsilon)^{\eta} \\
& =v^{m \tau-\tau} \epsilon^{-m \tau+\tau} q_{\gamma}(m \tau-\tau) \sum_{\varepsilon \in E} \epsilon^{\varepsilon}
\end{aligned}
$$

for some $\tau \in \mathbb{R}$. The claim follows, due to

$$
\sum_{\varepsilon \in E} \epsilon^{\varepsilon}= \begin{cases}m, & \epsilon=1 \\ 0, & \text { otherwise. }\end{cases}
$$

\subsection{Multivariate case}

In this subsection we give a closer look at the multivariate case. We will not repeat results that can be easily extended from the univariate setting by simply replacing $\mathbb{Z}, \mathbb{R}$ or $\mathbb{C}$ with $\mathbb{Z}^{s}, \mathbb{R}^{s}$ or $\mathbb{C}^{s}$, respectively. This is the case of Proposition 4.5 which we give without proof.

Recall that $m=|\operatorname{det}(M)|$ determines the cardinality of $E=\left\{\boldsymbol{\varepsilon}_{0}, \ldots, \boldsymbol{\varepsilon}_{m-1}\right\}$ and of $\Xi=\left\{e^{2 \pi i M^{-T} \boldsymbol{\xi}}: \boldsymbol{\xi}\right.$ is a coset representative of $\left.M^{-T} \mathbb{Z}^{s} / \mathbb{Z}^{s}\right\}$.

Proposition 4.5. A subdivision scheme defined by the symbols $\left\{a^{[k]}(\boldsymbol{z}), k \geq 0\right\}$ is $E P_{\Gamma, \Lambda}$-generating if and only if

$$
\begin{gathered}
D^{\gamma} a^{[k]}(\mathbf{v})=0, \quad \mathbf{v} \in V_{k}^{\prime}, \quad \gamma \in \Gamma, \quad k \geq 0, \\
\text { for } V_{k}^{\prime}=\left\{\left(v_{1}, \ldots, v_{s}\right)^{T}: v_{j}=\epsilon_{j} e^{-\left(\boldsymbol{\lambda} \cdot M^{-(k+1)}\right)_{j}}, \quad \boldsymbol{\lambda} \in \Lambda, \quad \boldsymbol{\epsilon} \in \Xi \backslash\{\mathbf{1}\}\right\} .
\end{gathered}
$$

\subsubsection{Reproduction of multivariate exponential polynomials}

The multivariate extension of Proposition 4.3 can appear trivial after reading the proof of Proposition 4.6. We believe that would not be the case, if we omitted its proof. There are also several crucial differences between the proofs of Theorem 4.7 and its univariate counterpart.

Proposition 4.6. A subdivision scheme $S_{\left\{\mathbf{a}^{[k]}, k \geq 0\right\}}$ is step-wise $E P_{\Gamma, \Lambda}$-reproducing if and only if there exists a shift parameter $\boldsymbol{\tau} \in \overline{\mathbb{R}}^{s}$ such that

$$
\sum_{\boldsymbol{\beta} \in \mathbb{Z}^{s}} \mathrm{a}_{M \boldsymbol{\beta}+\boldsymbol{\varepsilon}}^{[k]}\left(M^{-k} \boldsymbol{\beta}\right)^{\boldsymbol{\gamma}^{\prime}} \mathbf{v}^{M \boldsymbol{\beta}+\varepsilon}=\mathbf{v}^{M \boldsymbol{\tau}-\boldsymbol{\tau}} \boldsymbol{\epsilon}^{-M \boldsymbol{\tau}+\boldsymbol{\tau}+\varepsilon}\left(M^{-(k+1)}(M \boldsymbol{\tau}-\boldsymbol{\tau}-\boldsymbol{\varepsilon})\right)^{\boldsymbol{\gamma}^{\prime}}
$$

is satisfied for all $\mathbf{v} \in V_{k}=\left\{\left(v_{1}, \ldots, v_{s}\right)^{T}: v_{j}=\epsilon_{j} e^{-\left(\boldsymbol{\lambda} \cdot M^{-(k+1)}\right)_{j}}, \boldsymbol{\lambda} \in \Lambda, \boldsymbol{\epsilon} \in\right.$ $\Xi\}, k \geq 0, \gamma^{\prime} \leq \gamma, \gamma \in \Gamma, \varepsilon \in E$ and $\epsilon \in \Xi$. 
Proof: Let $\gamma \in \Gamma, \lambda \in \Lambda$ and define

$$
f_{\boldsymbol{\alpha}}^{[k]}=\left(M^{-k}(\boldsymbol{\alpha}+\boldsymbol{\tau})\right)^{\boldsymbol{\gamma}} \cdot e^{\boldsymbol{\lambda} \cdot M^{-k}(\boldsymbol{\alpha}+\boldsymbol{\tau})}, \quad \boldsymbol{\alpha} \in \mathbb{Z}^{s}, \quad k \geq 0 .
$$

By definition, the step-wise reproduction of sequences sampled from the exponentialpolynomials in $E P_{\Gamma, \Lambda}$ is equivalent to the existence of $\tau \in \mathbb{R}^{s}$ such that

$$
f_{M \boldsymbol{\alpha}+\varepsilon}^{[k+1]}=\sum_{\boldsymbol{\beta} \in \mathbb{Z}^{s}} \mathrm{a}_{M \boldsymbol{\beta}+\varepsilon}^{[k]} f_{\boldsymbol{\alpha}-\boldsymbol{\beta}}^{[k]}, \quad \varepsilon \in E, \quad \boldsymbol{\alpha} \in \mathbb{Z}^{s} .
$$

Multiplying both sides of (4.16) by $e^{-\boldsymbol{\lambda} \cdot M^{-(k+1)}(M \boldsymbol{\alpha}+M \boldsymbol{\tau}+\boldsymbol{\varepsilon})}$ we get

$$
\begin{aligned}
& \sum_{\boldsymbol{\beta} \in \mathbb{Z}^{s}} \mathrm{a}_{M \boldsymbol{\beta}+\boldsymbol{\varepsilon}}^{[k]}\left(M^{-k}(\boldsymbol{\alpha}-\boldsymbol{\beta}+\boldsymbol{\tau})\right)^{\boldsymbol{\gamma}} e^{-\boldsymbol{\lambda} \cdot M^{-(k+1)}(M \boldsymbol{\beta}+\boldsymbol{\varepsilon})} \\
&=\left(M^{-(k+1)}(M \boldsymbol{\alpha}+\boldsymbol{\varepsilon}+\boldsymbol{\tau})\right)^{\boldsymbol{\gamma}} e^{-\boldsymbol{\lambda} \cdot M^{-(k+1)}(M \boldsymbol{\tau}-\boldsymbol{\tau})},
\end{aligned}
$$

or, equivalently,

$$
\begin{aligned}
\sum_{\boldsymbol{\beta} \in \mathbb{Z}^{s}} \mathrm{a}_{M \boldsymbol{\beta}+\boldsymbol{\varepsilon}}^{[k]} & \left(M^{-k}(\boldsymbol{\alpha}-\boldsymbol{\beta}+\boldsymbol{\tau})\right)^{\boldsymbol{\gamma}} e^{\left(-\boldsymbol{\lambda} \cdot M^{-(k+1)}-2 \pi i M^{-T} \boldsymbol{\xi}\right) \cdot(M \boldsymbol{\beta}+\boldsymbol{\varepsilon})} \\
& =e^{-2 \pi i M^{-T} \boldsymbol{\xi} \cdot(M \boldsymbol{\beta}+\boldsymbol{\varepsilon}-M \boldsymbol{\tau}+\boldsymbol{\tau})}\left(M^{-(k+1)}(M \boldsymbol{\alpha}+\boldsymbol{\varepsilon}+\boldsymbol{\tau})\right)^{\boldsymbol{\gamma}} e^{\left(-\boldsymbol{\lambda} \cdot M^{-(k+1)}-2 \pi i M^{-T} \boldsymbol{\xi}\right) \cdot(M \boldsymbol{\tau}-\boldsymbol{\tau})} .
\end{aligned}
$$

Now, by properties of scalar products we have $M^{-T} \boldsymbol{\xi} \cdot M \boldsymbol{\beta}=\boldsymbol{\xi} \cdot M^{-1} M \boldsymbol{\beta}=$ $\boldsymbol{\xi} \cdot \boldsymbol{\beta} \in \mathbb{Z}$, and therefore $e^{-2 \pi i M^{-T} \boldsymbol{\xi} \cdot M \boldsymbol{\beta}}=1$. Thus, (4.17) is equivalent to

$$
\begin{aligned}
\sum_{\boldsymbol{\beta} \in \mathbb{Z}^{s}} \mathrm{a}_{M \boldsymbol{\beta}+\boldsymbol{\varepsilon}}^{[k]} & \left(M^{-k}(\boldsymbol{\alpha}-\boldsymbol{\beta}+\boldsymbol{\tau})\right)^{\boldsymbol{\gamma}} \mathbf{v}^{M \boldsymbol{\beta}+\boldsymbol{\varepsilon}} \\
& =\boldsymbol{\epsilon}^{-M \boldsymbol{\tau}+\boldsymbol{\tau}+\boldsymbol{\varepsilon}}\left(M^{-(k+1)}(M \boldsymbol{\alpha}+\boldsymbol{\varepsilon}+\boldsymbol{\tau})\right)^{\boldsymbol{\gamma}} \mathbf{v}^{M \boldsymbol{\tau}-\boldsymbol{\tau}},
\end{aligned}
$$

for $\mathbf{v} \in V_{k}$. Considering both sides of (4.18) as polynomials in $M^{-k} \boldsymbol{\alpha}$ with

$$
\left(M^{-k}(\boldsymbol{\alpha}-\boldsymbol{\beta}+\boldsymbol{\tau})\right)^{\boldsymbol{\gamma}}=\sum_{\eta \leq \boldsymbol{\gamma}}\left(\begin{array}{c}
\boldsymbol{\gamma} \\
\eta
\end{array}\right)\left(M^{-k}(-\boldsymbol{\beta}+\boldsymbol{\tau})\right)^{\eta}\left(M^{-k} \boldsymbol{\alpha}\right)^{\boldsymbol{\gamma}-\eta}
$$

and

$$
\left(M^{-(k+1)}(M \boldsymbol{\alpha}+\varepsilon+\boldsymbol{\tau})\right)^{\gamma}=\sum_{\eta \leq \gamma}\left(\begin{array}{l}
\gamma \\
\eta
\end{array}\right)\left(M^{-(k+1)}(\varepsilon+\boldsymbol{\tau})\right)^{\eta}\left(M^{-k} \boldsymbol{\alpha}\right)^{\gamma-\eta}
$$

we get that (4.18) is equivalent to

$$
\begin{aligned}
\sum_{\boldsymbol{\beta} \in \mathbb{Z}^{s}} \mathrm{a}_{M \boldsymbol{\beta}+\boldsymbol{\varepsilon}}^{[k]}\left(M^{-k}(-\boldsymbol{\beta}+\boldsymbol{\tau})\right)^{\eta} \mathbf{v}^{M \boldsymbol{\beta}+\boldsymbol{\varepsilon}} & \\
& =\boldsymbol{\epsilon}^{-M \boldsymbol{\tau}+\boldsymbol{\tau}+\boldsymbol{\varepsilon}}\left(M^{-(k+1)}(\boldsymbol{\varepsilon}+\boldsymbol{\tau})\right)^{\eta} \mathbf{v}^{M \boldsymbol{\tau}-\boldsymbol{\tau}}, \quad \mathbf{v} \in V_{k}, \quad \eta \leq \boldsymbol{\gamma} .
\end{aligned}
$$


Next, we expand

$$
\left(M^{-(k+1)}(M \boldsymbol{\tau}+\boldsymbol{\tau}-M \boldsymbol{\tau}+\varepsilon)\right)^{\eta}=\sum_{\gamma^{\prime} \leq \eta}\left(\begin{array}{c}
\eta \\
\gamma^{\prime}
\end{array}\right)\left(M^{-(k+1)}(\boldsymbol{\tau}-M \boldsymbol{\tau}+\varepsilon)\right)^{\gamma^{\prime}}\left(M^{-k} \boldsymbol{\tau}\right)^{\eta-\gamma^{\prime}}
$$

and, similarly, $\left(M^{-k}(-\boldsymbol{\beta}+\boldsymbol{\tau})\right)^{\eta}$. Thus, (4.19) is satisfied if and only if

$$
\begin{aligned}
& \sum_{\boldsymbol{\beta} \in \mathbb{Z}^{s}} \mathrm{a}_{M \boldsymbol{\beta}+\boldsymbol{\varepsilon}}^{[k]}\left(-M^{-k} \boldsymbol{\beta}\right)^{\boldsymbol{\gamma}^{\prime}} \mathbf{v}^{M \boldsymbol{\beta}+\boldsymbol{\varepsilon}}= \\
& \boldsymbol{\epsilon}^{-M \boldsymbol{\tau}+\boldsymbol{\tau}+\boldsymbol{\varepsilon}}\left(M^{-(k+1)}(\boldsymbol{\tau}+\boldsymbol{\varepsilon}-M \boldsymbol{\tau})\right)^{\boldsymbol{\gamma}^{\prime}} \mathbf{v}^{M \boldsymbol{\tau}-\boldsymbol{\tau}}, \quad \mathbf{v} \in V_{k}, \quad \boldsymbol{\gamma}^{\prime} \leq \eta \leq \boldsymbol{\gamma} .
\end{aligned}
$$

Multiplying both sides by $(-1)^{\left|\gamma^{\prime}\right|}$, we get the claim.

Define

$$
q_{\mathbf{0}}\left(z_{1}, \ldots, z_{s}\right)=1, \quad q_{\boldsymbol{\gamma}}\left(z_{1}, \ldots, z_{s}\right)=\prod_{\ell=1}^{s} \prod_{j=0}^{\gamma_{\ell}-1}\left(z_{\ell}-j\right), \quad \gamma \in \mathbb{N}_{0}^{s} .
$$

Theorem 4.7. A non-singular subdivision scheme $S_{\left\{\mathbf{a}^{[k]}, k \geq 0\right\}}$ reproduces $E P_{\Gamma, \Lambda}$ if and only if there exists a shift parameter $\boldsymbol{\tau} \in \mathbb{R}^{s}$ such that

$$
\mathbf{v}^{\gamma} D^{\gamma} a^{[k]}(\mathbf{v})= \begin{cases}m \cdot \mathbf{v}^{M \tau-\tau} q_{\boldsymbol{\gamma}}(M \boldsymbol{\tau}-\boldsymbol{\tau}), & \text { for all } \mathbf{v} \text { such that } \boldsymbol{\epsilon}=\mathbf{1}, \\ 0, & \text { otherwise }\end{cases}
$$

for all $\mathbf{v} \in V_{k}=\left\{\left(v_{1}, \ldots, v_{s}\right)^{T}: v_{j}=\epsilon_{j} e^{-\left(\boldsymbol{\lambda} \cdot M^{-(k+1)}\right)_{j}}, \boldsymbol{\lambda} \in \Lambda, \boldsymbol{\epsilon} \in \Xi\right\}, k \geq 0$, $\gamma \in \Gamma$.

Proof: Due to Proposition 3.6 it suffices to prove this statement for step-wise reproduction of sequences sampled from elements of $E P_{\Gamma, \Lambda}$. We use Proposition 4.6 and show that the step-wise $E P_{\Gamma, \Lambda}$-reproduction is equivalent to the identities (4.21). Consider $q_{\boldsymbol{\gamma}}(M \boldsymbol{\alpha}+\boldsymbol{\varepsilon})=q_{\boldsymbol{\gamma}}\left(M^{k+1} M^{-k} \boldsymbol{\alpha}+\boldsymbol{\varepsilon}\right)$, for fixed $\boldsymbol{\gamma} \in \Gamma$ and $\varepsilon \in E$, as a polynomial in $M^{-k} \boldsymbol{\alpha}, \boldsymbol{\alpha} \in \mathbb{Z}^{s}$, and write

$$
q_{\boldsymbol{\gamma}}\left(M^{k+1} M^{-k} \boldsymbol{\alpha}+\boldsymbol{\varepsilon}\right)=\sum_{\eta \leq \boldsymbol{\gamma}} c_{\boldsymbol{\gamma}, \boldsymbol{\varepsilon}, \eta}\left(M^{-k} \boldsymbol{\alpha}\right)^{\eta}, \quad c_{\boldsymbol{\gamma}, \boldsymbol{\varepsilon}, \eta} \in \mathbb{R}
$$

Let $\mathbf{v} \in V_{k}$. By Proposition 4.6 and by (4.22), the step-wise reproduction is equivalent to

$$
\begin{aligned}
\mathbf{v}^{\boldsymbol{\gamma}} D^{\boldsymbol{\gamma}} a^{[k]}(\mathbf{v}) & =\sum_{\boldsymbol{\varepsilon} \in E} \sum_{\boldsymbol{\alpha} \in \mathbb{Z}^{s}} \mathrm{a}_{M \boldsymbol{\alpha}+\boldsymbol{\varepsilon}}^{[k]} q_{\boldsymbol{\gamma}}(M \boldsymbol{\alpha}+\boldsymbol{\varepsilon}) \mathbf{v}^{M \boldsymbol{\alpha}+\boldsymbol{\varepsilon}} \\
& =\sum_{\boldsymbol{\varepsilon} \in E} \sum_{\eta \leq \boldsymbol{\gamma}} c_{\boldsymbol{\gamma}, \boldsymbol{\varepsilon}, \eta} \sum_{\boldsymbol{\alpha} \in \mathbb{Z}^{s}} \mathrm{a}_{M \boldsymbol{\alpha}+\boldsymbol{\varepsilon}}^{[k]}\left(M^{-k} \boldsymbol{\alpha}\right)^{\eta} \mathbf{v}^{M \boldsymbol{\alpha}+\boldsymbol{\varepsilon}} \\
& =\sum_{\boldsymbol{\varepsilon} \in E} \sum_{\eta \leq \boldsymbol{\gamma}} c_{\boldsymbol{\gamma}, \boldsymbol{\varepsilon}, \eta}\left(M^{-(k+1)}(M \boldsymbol{\tau}-\boldsymbol{\tau}-\boldsymbol{\varepsilon})\right)^{\eta} \mathbf{v}^{M \boldsymbol{\tau}-\boldsymbol{\tau}} \boldsymbol{\epsilon}^{-M \boldsymbol{\tau}+\boldsymbol{\tau}+\boldsymbol{\varepsilon}} \\
& =\mathbf{v}^{M \boldsymbol{\tau}-\boldsymbol{\tau}} \boldsymbol{\epsilon}^{-M \boldsymbol{\tau}+\boldsymbol{\tau}} q_{\boldsymbol{\gamma}}(M \boldsymbol{\tau}-\boldsymbol{\tau}) \sum_{\boldsymbol{\varepsilon} \in E} \boldsymbol{\epsilon}^{\boldsymbol{\varepsilon}}
\end{aligned}
$$


for some $\tau \in \mathbb{R}^{s}$. The claim follows due to

$$
\sum_{\varepsilon \in E} \boldsymbol{\epsilon}^{\varepsilon}= \begin{cases}m, & \boldsymbol{\epsilon}=\mathbf{1} \\ 0, & \text { otherwise. }\end{cases}
$$

In the case the scheme $S_{\left\{\mathbf{a}^{[k]}, k \geq 0\right\}}$ is convergent, but not non-singular, the conditions (4.21) in Theorem 4.7 are only sufficient for the $E P_{\Gamma, \Lambda}$-reproduction. Subdivision schemes generating exponential box splines, whose translates are linearly dependent, are important examples of such convergent schemes. See [34, Theorem 4.3] for conditions that characterize the linear independence of exponential box splines.

Corollary 4.8. A convergent subdivision scheme $S_{\left\{\mathbf{a}^{[k]}, k \geq 0\right\}}$ reproduces $E P_{\Gamma, \Lambda}$ if there exists a shift parameter $\boldsymbol{\tau} \in \mathbb{R}^{s}$ such that

$$
\mathbf{v}^{\gamma} D^{\gamma} a^{[k]}(\mathbf{v})= \begin{cases}m \cdot \mathbf{v}^{M \tau-\tau} q_{\boldsymbol{\gamma}}(M \boldsymbol{\tau}-\boldsymbol{\tau}), & \text { for all } \mathbf{v} \text { such that } \boldsymbol{\epsilon}=\mathbf{1}, \\ 0, & \text { otherwise, }\end{cases}
$$

for all $\mathbf{v} \in\left\{\left(v_{1}, \ldots, v_{s}\right)^{T}: v_{j}=\epsilon_{j} e^{-\left(\boldsymbol{\lambda} \cdot M^{-(k+1)}\right)_{j}}, \boldsymbol{\lambda} \in \Lambda, \boldsymbol{\epsilon} \in \Xi\right\}, k \geq 0, \gamma \in \Gamma$.

\section{Examples and applications}

\subsection{Shift factors and interpolatory schemes}

The first important application of Theorem 4.7, as in the binary univariate non-stationary case or as in the stationary multivariate case, is the analysis of the reproduction properties of interpolatory schemes, i.e. the schemes whose masks satisfy

$$
\mathrm{a}_{M \boldsymbol{\alpha}}^{[k]}=\delta_{\boldsymbol{\alpha}, 0}, \boldsymbol{\alpha} \in \mathbb{Z}^{s} .
$$

It is also of importance to analyse the effect of the mask shifts on the reproduction properties of the corresponding schemes. The following results are easily obtained by combining [12, Corollary 2] and [7, Proposition 3.4] or [12, Proposition 3] and [7, Lemma 3.1], respectively.

Corollary 5.1. A non-singular interpolatory subdivision scheme $S_{\left\{\mathbf{a}^{[k]}, k \geq 0\right\}}$ is $E P_{\Gamma, \Lambda}$-reproducing only if $\boldsymbol{\tau}=\mathbf{0}$. Moreover, the generation of $E P_{\Gamma, \Lambda}$ implies that the scheme reproduces the same space $E P_{\Gamma, \Lambda}$.

Corollary 5.2. If a non-singular subdivision scheme $S_{\left\{\mathbf{a}^{(k)}, k \geq 0\right\}}$ reproduces $E P_{\Gamma, \Lambda}$, then so does the scheme $S_{\left\{\mathbf{b}^{(k)}, k \geq 0\right\}}$ defined by the symbols $b^{(k)}(\boldsymbol{z})=$ $\boldsymbol{z}^{\boldsymbol{\beta}} \cdot a^{(k)}(\boldsymbol{z}), k \geq 0$ and $\boldsymbol{\beta} \in \mathbb{Z}^{s}$. 


\subsection{Subdivision schemes for exponential B-splines and exponential box splines}

In this subsection we study the reproduction properties of the subdivision schemes associated with the so-called exponential B-splines and box splines. The symbols of these schemes even in the non-stationary case play a role of smoothing factors [15]. They also determine the generation properties of subdivision schemes.

\subsubsection{Exponential B-splines}

It is well known that in the binary case a non-stationary subdivision scheme generates univariate exponential polynomials

$$
p(x)=x^{\gamma} e^{\lambda x} \quad \lambda \in \mathbb{C}, \quad \gamma \in \mathbb{N}_{0},
$$

if its symbol contains factors of the type

$$
\left(1+r_{k} z\right) \text { with a suitable } r_{k} \in \mathbb{C} \text {. }
$$

We show that in the $m$-ary case such exponential polynomials $p$ are generated by non-stationary subdivision schemes with symbols containing factors of the type

$$
\left(1+r_{k} z+r_{k}^{2} z^{2}+\cdots+r_{k}^{m-1} z^{m-1}\right) \quad \text { with a suitable } r_{k} \in \mathbb{C} .
$$

The following result is a generalization of a result in [15]. We present it here as it also allows for derivation of masks of exponential box splines in the next subsection and illustrates the generation property of the corresponding schemes.

Proposition 5.3. Let a non-stationary $m$-ary subdivision scheme be given by

$a^{[k]}(z)=\left(1+r_{k} z+r_{k}^{2} z^{2}+\cdots+r_{k}^{m-1} z^{m-1}\right), r_{k}=e^{\lambda m^{-(k+1)}}, \lambda \in \mathbb{C}, \quad k \geq 0$,

then its basic limit function is $\phi(x)=e^{\lambda x} \chi_{[0,1)}$.

Proof: First we observe that a subdivision scheme based on the masks (5.2) with support size $N_{k}=m$, whenever convergent or weakly convergent, generates a basic limit function supported on $\left[0, \frac{N_{k}-1}{m-1}\right]=[0,1]$, see for example [9]. The subdivision rules corresponding to the symbols in (5.2) are given by

$$
f_{m \alpha+\varepsilon}^{k+1}=r_{k}^{\varepsilon} f_{\alpha}^{k}, \quad \varepsilon \in E .
$$

Starting the subdivision process with $\boldsymbol{\delta}$, from (5.3) we get that the value of the basic limit function $\phi$ at any $m$-adic point

$$
x=\sum_{j=1}^{k} m^{-j} \varepsilon_{j}, \quad \varepsilon_{j} \in E,
$$

is

$$
\phi(x)=\prod_{j=1}^{k} r_{j-1}^{\varepsilon_{j}} .
$$


At every non $m$-adic point $x=\sum_{j=1}^{\infty} m^{-j} \varepsilon_{j}, \varepsilon_{j} \in E$, we can define

$$
\phi(x)=\prod_{j=1}^{\infty} r_{j-1}^{\varepsilon_{j}}
$$

due to $\sum_{k \in \mathbb{Z}_{+}}\left|1-r_{k}\right|<\infty$, which ensures the convergence of this infinite product.

For the point $x \notin[0,1)$ we set $\phi(x)=0$. To show the continuity of $\phi$ at non $m$-adic points we consider a sequence of points

$$
\left\{x^{\ell}, \ell \in \mathbb{N}_{0}\right\}, \quad \text { with } \quad x^{\ell}=\sum_{j=1}^{k_{\ell}} m^{-j} \varepsilon_{j}+\sum_{j=k_{\ell}+1}^{\infty} m^{-j} \varepsilon_{j}^{\ell}, \quad \varepsilon_{j}^{\ell} \in E,
$$

such that $k_{\ell}$ goes to infinity as $\ell$ goes to infinity. Then

$$
\lim _{\ell \rightarrow \infty}\left|\phi\left(x^{\ell}\right)-\phi(x)\right|=\lim _{\ell \rightarrow \infty}\left|\prod_{j=1}^{\infty} r_{j-1}^{\varepsilon_{j}}-\prod_{j=1}^{k_{\ell}} r_{j-1}^{\varepsilon_{j}} \prod_{j=k_{\ell}+1}^{\infty} r_{j-1}^{\varepsilon_{j}^{\ell}}\right|=0 .
$$

The non-uniqueness of the representations of $m$-adic points

$$
x=\sum_{j=1}^{k} m^{-j} \varepsilon_{j}=\sum_{j=1}^{k-1} m^{-j} \varepsilon_{j}+m^{-k}\left(\varepsilon_{k}-1\right)+\sum_{j=k+1}^{\infty} m^{-j}(m-1)
$$

with

$$
\sum_{j=k+1}^{\infty} m^{-j}(m-1)=m^{-k}
$$

makes the analysis of continuity at these points more involved. Thus, we need to consider two types of sequences that converge to $x$ from the left and from the right. This implies that $\phi$ is continuous at $m$-adic points if and only if

$$
r_{0}^{\varepsilon_{1}} \ldots r_{k-2}^{\varepsilon_{k-1}}\left(r_{k-1}^{\varepsilon_{k}}-r_{k-1}^{\varepsilon_{k}-1} \prod_{k+1}^{\infty} r_{j-1}^{m-1}\right)=0
$$

or, equivalently,

$$
r_{k-1}=\prod_{j=k+1}^{\infty} r_{j-1}^{m-1}=r_{k}^{m-1} \prod_{j=k+2}^{\infty} r_{j-1}^{m-1}=r_{k}^{m} \quad \Leftrightarrow \quad r_{k}=r_{k-1}^{1 / m}=\cdots=r_{0}^{1 / m^{k}} .
$$

Hence, for $r_{0}=e^{\lambda / m}, \lambda \in \mathbb{C}$, and $r_{k}=e^{\lambda / m^{k+1}}$ we get

$$
\phi(x)=\prod_{j=1}^{\infty} r_{j-1}^{\varepsilon_{j}}=\prod_{j=1}^{\infty} r_{0}^{\varepsilon_{j} m^{-(j+1)}}=\prod_{j=1}^{\infty}\left(e^{\lambda \varepsilon_{j} m^{-j}}\right)=e^{\lambda \sum_{j=1}^{\infty} m^{-j} \varepsilon_{j}}=e^{\lambda x} .
$$


Proposition 5.3 implies that the corresponding subdivision scheme generates the exponential polynomials $e^{\lambda x}$. Next, we use the algebraic conditions on the subdivision symbols in Theorem 4.4 to show how to normalize the symbols appropriately to ensure the reproduction of exponential polynomials $e^{\lambda x}$.

Lemma 5.4. Let $\Gamma=\{0\}$ and $\Lambda=\{\lambda\}, \lambda \in \mathbb{C}$. Then a non-singular nonstationary scheme given by

$$
a^{[k]}(z)=K^{[k]}\left(1+r_{k} z+r_{k}^{2} z^{2}+\cdots+r_{k}^{m-1} z^{m-1}\right), r_{k}=e^{\lambda m^{-(k+1)}}, k \geq 0,
$$

reproduces $E P_{\Gamma, \Lambda}=\left\{e^{\lambda x}\right\}$ if and only if $K^{[k]}=r_{k}^{-m \tau+\tau}$ and $\tau \in \mathbb{R}$.

Proof: In this case

$$
V_{k}=\left\{e^{2 \pi i \varepsilon / m} e^{-\lambda m^{-(k+1)}}: \varepsilon \in E\right\}, \quad k \geq 0 .
$$

We only need to check the algebraic condition $a^{[k]}(v)=m v^{m \tau-\tau}$ for $v=r_{k}^{-1}=$ $e^{-\lambda m^{-(k+1)}}$ as the rest of the conditions in (4.13) are trivially satisfied. By Theorem 4.4 the scheme reproduces $E P_{\Gamma, \Lambda}$ if and only if for all $k \geq 0$ we have

$$
K^{[k]} m=a^{[k]}\left(r_{k}^{-1}\right)=m r_{k}^{-m \tau+\tau} .
$$

All these identities are satisfied for $\tau \in \mathbb{R}$ and $K^{[k]}=r_{k}^{-m \tau+\tau}$.

In the following remark we list several important properties of the exponential B-splines.

Remark 5.5. (i) The scheme associated with

$$
a^{[k]}(z)=\left(\sum_{\varepsilon \in E} r_{k}^{\varepsilon} z^{\varepsilon}\right)\left(\sum_{\varepsilon \in E} s_{k}^{\varepsilon} z^{\varepsilon}\right)
$$

for $r_{k}=e^{\lambda m^{-(k+1)}}$ and $s_{k}=e^{\mu m^{-(k+1)}}, \lambda, \mu \in \mathbb{C}, k \geq 0$, is convergent, [15, [16]. It has the basic limit function $\phi=\phi_{1} * \phi_{2}$ with $\phi_{1}(x):=e^{\lambda x} \chi_{[0,1)}$ and $\phi_{2}(x)=$ $e^{\mu x} \chi_{[0,1)}$. The function $\phi_{1} * \phi_{2}$ is $C^{0}$, locally an exponential function on $[0,1)$ and $[1,2)$, globally supported on $[0,2)$ and is a linear combination of $e^{\lambda x}$ and $e^{\mu x}$.

(ii) In general, the $n$-fold convolution $\phi=\beta_{1} * \beta_{2} * \cdots * \beta_{n}$ is $C^{n-2}$, locally an exponential function on $[J-1, J), J=1, \ldots, n$, globally supported on $[0, n)$ and is a linear combination of the corresponding exponential functions. Such a function $\phi$ is a basic limit function of the non-stationary scheme given by $n$-fold products of the symbols in (5.2). Thus, exponential polynomials are generated by non-stationary subdivision schemes with factors in (5.2).

(iii) By [34, Theorem 4.3] and the compact support of the masks, the exponential B-splines satisfy the assumptions of Proposition 2.2, if no two purely imaginary $\lambda$ and $\mu$ satisfy $m^{-\ell}(\lambda-\mu)=2 \pi i \ell$ for $\ell \geq L, L \geq 0$. Thus, the corresponding non-stationary schemes are non-singular. 
In the case of several exponential factors the space $E P_{\Gamma, \Lambda}$ that is reproduced by the associated non-stationary subdivision scheme is at most of dimension 2 . The results of Propositions 5.6 and 5.7 are consistent with the observation in [24] for the case $m=2$.

Proposition 5.6. Let $\Gamma=\{0\}$ and $\Lambda=\{\lambda\}, \lambda \in \mathbb{C}$. Then the non-singular non-stationary scheme defined by

$$
a^{[k]}(z)=K^{[k]}\left(1+r_{k} z+r_{k}^{2} z^{2}+\cdots+r_{k}^{m-1} z^{m-1}\right)^{n}, r_{k}=e^{\lambda m^{-(k+1)}}, n \geq 2, \text { (5.6) }
$$

reproduces at most $E P_{\Gamma, \Lambda}=\left\{e^{\lambda x}, x e^{\lambda x}\right\}$, if $K^{[k]}=m^{1-n} r_{k}^{-m \tau+\tau}$ and $\tau \in \mathbb{R}$.

Proof: We only need to check the algebraic conditions $a^{[k]}(v)=m v^{m \tau-\tau}$ and $v{\frac{d a^{[k]}(z)}{d z}}_{z=v}=m v^{m \tau-\tau} \tau$ at $v=r_{k}^{-1}=e^{-\lambda m^{-(k+1)}}$ as the rest of the conditions in (4.13) are trivially satisfied. By Theorem 4.4 the scheme reproduces $E P_{\Gamma, \Lambda}$ if and only if

$$
\begin{aligned}
K^{[k]} m^{n} & =m r_{k}^{-m \tau+\tau} \\
r_{k}^{-1} K^{[k]} n r_{k} m^{n-1} \frac{m(m-1)}{2} & =m r_{k}^{-m \tau+\tau} \tau .
\end{aligned}
$$

The first two identities imply that $K^{[k]}=m^{1-n} r_{k}^{-m \tau+\tau}$ with $\tau=\frac{n(m-1)}{2}$. Next, to guarantee the reproduction of the exponential polynomial $x^{2} e^{\lambda x}$, the algebraic condition $v^{2}{\frac{d^{2} a^{[k]}(z)}{d z^{2}}}_{z=v}=m v^{m \tau-\tau} \tau(\tau-1)$ should be satisfied. Or, equivalently, we get

$r_{k}^{-2} K^{[k]}\left(n(n-1) r_{k}^{2} m^{n-2} \frac{(m-1)^{2} m^{2}}{4}+n m^{n-1} r_{k}^{2} \frac{1}{3} m(m-1)(m-2)\right)=m r_{k}^{-m \tau+\tau} \tau(\tau-1)$,

that becomes

$$
\frac{1}{4} n(n-1)(m-1)^{2}+\frac{1}{3} n(m-1)(m-2)=\frac{n(m-1)}{2}\left(\frac{n(m-1)}{2}-1\right),
$$

or, equivalently,

$$
3(n-1)(m-1)+4(m-2)=3 n(m-1)-6
$$

which can be only satisfied for $m=-1$.

We continue with the analysis of another case.

Proposition 5.7. Let $\Gamma=\{0\}$ and $\Lambda=\{\lambda, \mu\}, \lambda, \mu \in \mathbb{C}, \lambda \neq \mu$. Then the non-singular non-stationary scheme given by

$$
a^{[k]}(z)=K^{[k]}\left(\sum_{\varepsilon \in E} r_{k}^{\varepsilon} z^{\varepsilon}\right)^{n}\left(\sum_{\varepsilon \in E} s_{k}^{\varepsilon} z^{\varepsilon}\right)^{n}, \quad r_{k}=e^{\lambda m^{-(k+1)}}, \quad s_{k}=e^{\mu m^{-(k+1)}}
$$


reproduces at most $E P_{\Gamma, \Lambda}=\left\{e^{\lambda x}, e^{\mu x}\right\}$, if

$$
K^{[k]}=m^{1-n}\left(\sum_{\varepsilon \in E} r_{k}^{m-1-\varepsilon} s_{k}^{\varepsilon}\right)^{-n} \quad \text { and } \quad \tau=n .
$$

Proof: We only need to ensure that $a^{[k]}(v)=m v^{m \tau-\tau}$ for $v \in\left\{r_{k}^{-1}, s_{k}^{-1}\right\}$ as the rest of the conditions in (4.13) are trivially satisfied. By Theorem 4.4 the scheme reproduces $E P_{\Gamma, \Lambda}$ if and only if

$$
\begin{aligned}
& K^{[k]} m^{n}\left(\sum_{\varepsilon \in E} r_{k}^{-\varepsilon} s_{k}^{\varepsilon}\right)^{n}=m r_{k}^{-m \tau+\tau}, \\
& K^{[k]} m^{n}\left(\sum_{\varepsilon \in E} r_{k}^{\varepsilon} s_{k}^{-\varepsilon}\right)^{n}=m s_{k}^{-m \tau+\tau} .
\end{aligned}
$$

Therefore, the reproduction of $E P_{\Gamma, \Lambda}$ is possible if and only if

$$
\begin{aligned}
& K^{[k]} m^{n} r_{k}^{n(1-m)}\left(\sum_{\varepsilon \in E} r_{k}^{m-1-\varepsilon} s_{k}^{\varepsilon}\right)^{n}=m r_{k}^{-m \tau+\tau} \\
& K^{[k]} m^{n} s_{k}^{n(1-m)}\left(\sum_{\varepsilon \in E} r_{k}^{\varepsilon} s_{k}^{m-1-\varepsilon}\right)^{n}=m s_{k}^{-m \tau+\tau},
\end{aligned}
$$

or, equivalently,

$$
r_{k}^{(\tau-n)(1-m)}=s_{k}^{(\tau-n)(1-m)},
$$

which is the case if and only if either $\tau=n$ or $\lambda=\mu$. For $\tau=n$ the conditions on the first derivative of $a^{[k]}(z)$ at $z=r_{k}^{-1}$ are satisfied if and only if $\lambda=\mu$.

Remark 5.8. Increasing the number of different factors in (5.7) to 3 would lead to the system of equations

$$
\begin{aligned}
\left(\sum_{\varepsilon \in E} w_{k}^{m-1-\varepsilon} s_{k}^{\varepsilon}\right)^{n} r_{k}^{(\tau-2 n)(1-m)} & =\left(\sum_{\varepsilon \in E} w_{k}^{m-1-\varepsilon} r_{k}^{\varepsilon}\right)^{n} s_{k}^{(\tau-2 n)(1-m)} \\
& =\left(\sum_{\varepsilon \in E} r_{k}^{m-1-\varepsilon} s_{k}^{\varepsilon}\right)^{n} w_{k}^{(\tau-2 n)(1-m)}
\end{aligned}
$$

in four unknowns $\tau, r_{k}=e^{\lambda m^{-(k+1)}}, s_{k}=e^{\mu m^{-(k+1)}}$ and $w_{k}=e^{\eta m^{-(k+1)}}$. Numerical experiments show that these are satisfied when either $\lambda=\eta=\mu$ or only two of $\lambda, \eta, \mu$ are different. The analysis of other possible schemes is not one of our goals. 


\subsection{Exponential box splines}

In this subsection we describe the structure of the symbols of non-stationary schemes associated with exponential box splines. We use them in subsection 5.6 to define a non-stationary butterfly scheme.

Let $M=n I, n \geq 2$. Then $m=|\operatorname{det} M|=n^{s}$ and $E=\{0, n-1\}^{s}$.

Proposition 5.9. Let

$$
a^{[k]}(\boldsymbol{z})=\sum_{\varepsilon \in E} \mathbf{r}_{k}^{\varepsilon} \boldsymbol{z}^{\varepsilon}, \quad k \geq 0, \quad \mathbf{r}_{k}=e^{\boldsymbol{\lambda} \cdot M^{-k-1}}, \quad \boldsymbol{\lambda} \in \mathbb{C}^{s}
$$

Then the basic limit function is $\phi(\boldsymbol{x})=e^{\boldsymbol{\lambda} \cdot \boldsymbol{x}} \chi_{[0,1)^{s}}$.

Proof: As the symbols $a^{[k]}(\boldsymbol{z})$ have a tensor-product structure, the proof is a straightforward generalization of our univariate result on exponential B-splines. We only need to observe that (see e.g. [20]), even for general dilation matrices, every point $\boldsymbol{x}$ in the support of $\phi$ has the representation

$$
\boldsymbol{x}=\sum_{j=1}^{\infty} M^{-j} \boldsymbol{\varepsilon}_{j}, \quad \boldsymbol{\varepsilon}_{j} \in E .
$$

The difficulty of studying the continuity of $\phi$ arises only at the points having at least one $M$-adic component, i.e.,

$x_{\ell}=\sum_{j=1}^{k}\left(M^{-j} \varepsilon_{j}\right)_{\ell}=\sum_{j=1}^{k-1}\left(M^{-j} \varepsilon_{j}\right)_{\ell}+n^{-k}\left(\varepsilon_{k, \ell}-1\right)+\sum_{j=k+1}^{\infty} M^{-j}(n-1) \mathbf{1}_{\ell}, \varepsilon_{k, \ell} \neq 0$,

where $\mathbf{1}_{\ell}$ is the standard $\ell$-th unit vector of $\mathbb{R}^{s}$ and

$$
\begin{aligned}
\sum_{j=k+1}^{\infty} M^{-j}(n-1) \mathbf{1} & =M^{-k-1}\left(\sum_{j=0}^{\infty} M^{-j}\right)(n-1) \mathbf{1} \\
& =M^{-k-1}\left(I-M^{-1}\right)^{-1}(n-1) \mathbf{1} \\
& =M^{-k}(M-I)^{-1}(n-1) \mathbf{1}=M^{-k} \mathbf{1} .
\end{aligned}
$$

Remark 5.10. By [35, (4.1)] or by [15, example 6], the scheme defined by the masks in (5.8) converges weakly to an exponential box spline $e^{\boldsymbol{\lambda} \boldsymbol{x}} \chi_{[0,1)^{s}}$, where $\chi_{[0,1)^{s}}$ is a characteristic function of $[0,1)^{s}$. To get the similar result for $\phi$ with sets of directions $\Upsilon=\left\{v_{1}, \ldots v_{s}\right\}$, span $\Upsilon=\mathbb{R}^{s}$, other than $E$, one just carries out the affine transformation $\mathbf{y}=\left[v_{1} \ldots v_{s}\right] \boldsymbol{x}$ for $\boldsymbol{x} \in[0,1)^{s}$. See [15, example 6] for the masks of the smoother exponential box splines. 
5.4. A $C^{2}$ binary, non-stationary, dual 4-point subdivision scheme reproducing conics

In this and the following subsections we show that our algebraic conditions in (4.13) can be efficiently used for constructing new univariate subdivision schemes with desired reproduction properties and with enhanced smoothness.

We start by deriving the non-stationary counterpart of the binary dual 4point subdivision scheme in [17]. We show that, compared to the binary nonstationary interpolatory 4-point scheme in [1], our scheme reproduces the same space of exponential polynomials, i.e. $\operatorname{span}\left\{1, x, e^{\lambda x}, e^{-\lambda x}\right\}$ with $\lambda \in(\mathbb{R} \cup i \mathbb{R}) \backslash$ $\{0\}$, but it is $C^{2}$ instead of $C^{1}$. Furthermore, among all existing non-stationary binary schemes that are $C^{2}$ and reproduce conic sections, see [10, 11, 12, 18, 24, 25, 31] , our scheme turns out to be the one with the smallest support.

In order to define our scheme, we consider a sequence of masks of the form

$$
\mathbf{a}^{[k]}=\left\{\cdots, 0, c_{3,2}^{[k]}, c_{0,1}^{[k]}, c_{2,2}^{[k]}, c_{1,1}^{[k]}, c_{1,2}^{[k]}, c_{2,1}^{[k]}, c_{0,2}^{[k]}, c_{3,1}^{[k]}, 0 \cdots\right\}, \quad k \geq 0,
$$

and make use of Corollary 4.8 to determine $c_{i, j}^{[k]}, i=0, \ldots, 3, j=1,2$, and the shift parameter $\tau \in \mathbb{R}$ such that the space $\operatorname{span}\left\{1, x, e^{\lambda x}, e^{-\lambda x}\right\}$ is reproduced. Namely, let $\Lambda=\{0, \lambda,-\lambda\}, \lambda \in(\mathbb{R} \cup i \mathbb{R}) \backslash\{0\}$, and $\Gamma=\{0,1\}$. Then, for $E=\{0,1\}$ and $k \geq 0$, we have

$$
V_{k}=\left\{e^{\pi i \varepsilon} e^{-\lambda 2^{-(k+1)}}: \varepsilon \in E, \lambda \in \Lambda\right\}
$$

and

$$
V_{k}^{\prime}=\left\{-e^{-\lambda 2^{-(k+1)}}: \lambda \in \Lambda\right\}=\left\{-e^{\lambda 2^{-(k+1)}},-e^{-\lambda 2^{-(k+1)}},-1\right\} .
$$

Thus, by Corollary 4.8 and Remark 3.2 (ii), for $Q=\{(0,0),(1,0),(0, \lambda),(0,-\lambda)\}$, we need to solve the following linear system of 8 equations in 8 unknowns

$$
\left\{\begin{array}{l}
a^{[k]}(v)=0, \quad \forall v \in V_{k}^{\prime}, \\
a^{[k]}(v)=2 v^{\tau}, \quad \forall v \in\left\{e^{\lambda 2^{-(k+1)}}, e^{-\lambda 2^{-(k+1)}}, 1\right\}, \\
\left.\frac{d a^{[k]}(z)}{d z}\right|_{z=v}=0, \quad \text { for } v=-1, \\
\left.\frac{d a^{[k]}(z)}{d z}\right|_{z=v}=2 \tau, \quad \text { for } v=1 .
\end{array}\right.
$$

We get $\tau=-\frac{1}{2}$ and

$$
\begin{aligned}
c_{0,2}^{[k]}=c_{0,1}^{[k]} & =-\frac{6\left(w^{[k]}\right)^{2}+2 w^{[k]}-1}{64\left(w^{[k]}\right)^{3}\left(2\left(w^{[k]}\right)^{2}-1\right)\left(w^{[k]}+1\right)}, \\
c_{1,2}^{[k]}=c_{1,1}^{[k]} & =\frac{10\left(w^{[k]}\right)^{2}+2 w^{[k]}-3}{64\left(w^{[k]}\right)^{3}\left(2\left(w^{[k]}\right)^{2}-1\right)\left(w^{[k]}+1\right)}+\frac{3}{4}, \\
c_{2,2}^{[k]}=c_{2,1}^{[k]} & =\frac{-2\left(w^{[k]}\right)^{2}+2 w^{[k]}+3}{64\left(w^{[k]}\right)^{3}\left(2\left(w^{[k]}\right)^{2}-1\right)\left(w^{[k]}+1\right)}+\frac{1}{4}, \\
c_{3,2}^{[k]}=c_{3,1}^{[k]} & =-\frac{2\left(w^{[k]}\right)^{2}+2 w^{[k]}+1}{64\left(w^{[k]}\right)^{3}\left(2\left(w^{[k]}\right)^{2}-1\right)\left(w^{[k]}+1\right)},
\end{aligned}
$$


with

$$
w^{[k]}=\frac{1}{2}\left(e^{2^{-(k+1)} \lambda / 2}+e^{-2^{-(k+1)} \lambda / 2}\right) .
$$

In conclusion, the non-stationary dual 4-point subdivision scheme we propose is defined by the $k$-level symbol

$$
\begin{aligned}
& a^{[k]}(z)=-z^{-4} \frac{1}{64\left(w^{[k]}\right)^{3}\left(2\left(w^{[k]}\right)^{2}-1\right)\left(w^{[k]}+1\right)}(z+1)^{3}\left(z^{2}+\left(4\left(w^{[k]}\right)^{2}-2\right) z+1\right) . \\
& \cdot\left(\left(2\left(w^{[k]}\right)^{2}+2 w^{[k]}+1\right) z^{2}-\left(8\left(w^{[k]}\right)^{4}+8\left(w^{[k]}\right)^{3}+2\right) z+2\left(w^{[k]}\right)^{2}+2 w^{[k]}+1\right) .
\end{aligned}
$$

We observe that

$$
\lim _{k \rightarrow \infty} c_{0,1}^{[k]}=-\frac{7}{128}, \quad \lim _{k \rightarrow \infty} c_{1,1}^{[k]}=\frac{105}{128}, \quad \lim _{k \rightarrow \infty} c_{2,1}^{[k]}=\frac{35}{128}, \quad \lim _{k \rightarrow \infty} c_{3,1}^{[k]}=-\frac{5}{128}
$$

i.e. when $k$ tends to infinity $\mathbf{a}^{[k]}$ converges to the mask of the above mentioned stationary dual 4-point subdivision scheme. More precisely, the result in [15, Theorem 8], implies that our non-stationary scheme is asymptotically equivalent (of "order" 2) to the stationary dual 4-point subdivision scheme in [17]. This property allows us to conclude that our scheme is indeed $C^{2}$.

\subsection{A $C^{2}$ ternary, non-stationary, dual 4-point subdivision scheme reproducing conics}

In this subsection we derive a $C^{2}$ ternary, non-stationary, dual 4-point subdivision scheme reproducing the space $\operatorname{span}\left\{1, x, e^{\lambda x}, e^{-\lambda x}\right\}$ with $\lambda \in(\mathbb{R} \cup i \mathbb{R}) \backslash$ $\{0\}$. Compared with the ternary, non-stationary, interpolatory 4-point scheme in [2], our scheme will have an additional feature of reproducing conic sections. Compared with the ternary, non-stationary, interpolatory 4-point scheme that reproduces the same space of exponential polynomials and is $C^{1}[3,4]$, our scheme will be $C^{2}$.

As in the previous subsection, we start by defining a sequence of masks of the form

$\mathbf{a}^{[k]}=\left\{\cdots, 0, c_{0,3}^{[k]}, c_{0,2}^{[k]}, c_{0,1}^{[k]}, c_{1,3}^{[k]}, c_{1,2}^{[k]}, c_{1,1}^{[k]}, c_{2,3}^{[k]}, c_{2,2}^{[k]}, c_{2,1}^{[k]}, c_{3,3}^{[k]}, c_{3,2}^{[k]}, c_{3,1}^{[k]}, 0, \cdots\right\}$,

and we derive the coefficients $c_{i, j}^{[k]}, i=0, \ldots, 3, j=1,2,3$, and the shift parameter $\tau \in \mathbb{R}$ by requiring that the scheme reproduces $\operatorname{span}\left\{1, x, e^{\lambda x}, e^{-\lambda x}\right\}$. Let $\Lambda=\{0, \lambda,-\lambda\}, \lambda \in(\mathbb{R} \cup i \mathbb{R}) \backslash\{0\}$, and $\Gamma=\{0,1\}$. Then, for $E=\{0,1,2\}$ and $k \geq 0$, we have

$$
V_{k}=\left\{e^{\frac{2 \pi i \varepsilon}{3}} e^{-\lambda 3^{-(k+1)}}: \varepsilon \in E, \lambda \in \Lambda\right\}
$$

and

$$
V_{k}^{\prime}=\left\{e^{\frac{2 \pi i \varepsilon}{3}} e^{-\lambda 3^{-(k+1)}}: \varepsilon \in E \backslash\{0\}, \lambda \in \Lambda\right\}
$$


The corresponding algebraic conditions of Corollary 4.8 and Remark 3.2 (ii) with $Q=\{(0,0),(1,0),(0, \lambda),(0,-\lambda)\}$ lead to the following system of 12 equations in 12 unknown

$$
\left\{\begin{array}{l}
a^{[k]}(v)=0, \quad \forall v \in V_{k}^{\prime}, \\
a^{[k]}(v)=3 v^{2 \tau}, \quad \forall v \in\left\{e^{\lambda 3^{-(k+1)}}, e^{-\lambda 3^{-(k+1)}}, 1\right\} \\
\left.\frac{d a^{[k]}(z)}{d z}\right|_{z=v}=0, \quad v=e^{\frac{2 \pi i \varepsilon}{3}}, \quad \varepsilon=1,2 \\
\left.\frac{d a^{[k]}(z)}{d z}\right|_{z=v}=6 \tau \quad \text { for } v=1 .
\end{array}\right.
$$

Solving the above system we get $\tau=-\frac{1}{4}$,

$$
\begin{aligned}
c_{0,2}^{[k]} & =-\frac{1}{8 w^{[k]}\left(2 w^{[k]}-1\right)^{2}\left(4\left(w^{[k]}\right)^{2}-3\right)\left(w^{[k]}+1\right)}, \\
c_{1,2}^{[k]} & =\frac{1}{8 w^{[k]}\left(2 w^{[k]}-1\right)^{2}\left(4\left(w^{[k]}\right)^{2}-3\right)\left(w^{[k]}+1\right)}+\frac{1}{2}, \\
c_{2,2}^{[k]} & =\frac{1}{8 w^{[k]}\left(2 w^{[k]}-1\right)^{2}\left(4\left(w^{[k]}\right)^{2}-3\right)\left(w^{[k]}+1\right)}+\frac{1}{2}, \\
c_{3,2}^{[k]} & =-\frac{1}{8 w^{[k]}\left(2 w^{[k]}-1\right)^{2}\left(4\left(w^{[k]}\right)^{2}-3\right)\left(w^{[k]}+1\right)}
\end{aligned}
$$

and

$$
\begin{aligned}
c_{0,3}^{[k]}=c_{3,1}^{[k]} & =\frac{16\left(w^{[k]}\right)^{4}+16\left(w^{[k]}\right)^{3}+3}{24 w^{[k]}\left(4\left(w^{[k]}\right)^{2}-1\right)^{3}\left(-4\left(w^{[k]}\right)^{3}-4\left(w^{[k]}\right)^{2}+3 w^{[k]}+3\right)}, \\
c_{1,3}^{[k]}=c_{2,1}^{[k]} & =-\frac{16\left(w^{[k]}\right)^{4}-16\left(w^{[k]}\right)^{2}-4 w^{[k]}-1}{8 w^{[k]}\left(2 w^{[k]}-1\right)^{3}\left(2 w^{[k]}+1\right)^{3}\left(4\left(w^{[k]}\right)^{2}-3\right)\left(w^{[k]}+1\right)}+\frac{1}{6}, \\
c_{2,3}^{[k]}=c_{1,1}^{[k]} & =\frac{48\left(w^{[k]}\right)^{4}+16\left(w^{[k]}\right)^{3}-32\left(w^{[k]}\right)^{2}-8 w^{[k]}+1}{8 w^{[k]}\left(2 w^{[k]}-1\right)^{3}\left(2 w^{[k]}+1\right)^{3}\left(4\left(w^{[k]}\right)^{2}-3\right)\left(w^{[k]}+1\right)}+\frac{5}{6}, \\
c_{3,3}^{[k]}=c_{0,1}^{[k]} & =\frac{80\left(w^{[k]}\right)^{4}+32\left(w^{[k]}\right)^{3}-48\left(w^{[k]}\right)^{2}-12 w^{[k]}+3}{24 w^{[k]}\left(4\left(w^{[k]}\right)^{2}-1\right)^{3}\left(-4\left(w^{[k]}\right)^{3}-4\left(w^{[k]}\right)^{2}+3 w^{[k]}+3\right)}
\end{aligned}
$$

where

$$
w^{[k]}=\frac{1}{2}\left(e^{3^{-(k+1)} \lambda / 2}+e^{-3^{-(k+1)} \lambda / 2}\right) .
$$

The resulting $k$-level symbol is

$$
\begin{aligned}
& a^{[k]}(z)=-z^{-6} K^{[k]}\left(z^{2}+z+1\right)^{2}(z+1) \\
& \left(z^{4}+\left(4\left(w^{[k]}\right)^{2}-2\right) z^{3}+\left(16\left(w^{[k]}\right)^{4}-16\left(w^{[k]}\right)^{2}+3\right) z^{2}+\left(4\left(w^{[k]}\right)^{2}-2\right) z+1\right) . \\
& \left(\left(16\left(w^{[k]}\right)^{4}+16\left(w^{[k]}\right)^{3}+3\right) z^{2}+\left(-64\left(w^{[k]}\right)^{6}-64\left(w^{[k]}\right)^{5}+32\left(w^{[k]}\right)^{4}+\right.\right. \\
& \left.\left.32\left(w^{[k]}\right)^{3}-12\left(w^{[k]}\right)^{2}-12 w^{[k]}-6\right) z+16\left(w^{[k]}\right)^{4}+16\left(w^{[k]}\right)^{3}+3\right),
\end{aligned}
$$

where

$$
K^{[k]}=\frac{1}{24 w^{[k]}\left(2 w^{[k]}-1\right)^{3}\left(2 w^{[k]}+1\right)^{3}\left(4\left(w^{[k]}\right)^{2}-3\right)\left(w^{[k]}+1\right)} .
$$


This non-stationary dual 4-point scheme is "order" 2 asymptotically equivalent to the stationary dual 4-point scheme with the mask

$\lim _{k \rightarrow \infty} \mathbf{a}^{[k]}=\left\{-\frac{35}{1296},-\frac{1}{16},-\frac{55}{1296}, \frac{77}{432}, \frac{9}{16}, \frac{385}{432}, \frac{385}{432}, \frac{9}{16}, \frac{77}{432},-\frac{55}{1296},-\frac{1}{16},-\frac{35}{1296}\right\}$.

and its symbol satisfies

$$
\lim _{k \rightarrow \infty} a^{[k]}(z)=-z^{-6} \frac{1}{1296}\left(z^{2}+z+1\right)^{4}(z+1)\left(35 z^{2}-94 z+35\right) .
$$

By [13, Section 5.2], this stationary scheme is $C^{2}$. Thus, applying [15, Theorem 8] we deduce that our non-stationary scheme is also $C^{2}$.

The rest of this section is devoted to the multivariate case.

\subsection{Non-stationary butterfly scheme}

In this subsection, we derive a non-stationary butterfly scheme using the results in [8]. Let $\mathbf{r}_{k}=e^{\boldsymbol{\lambda} 2^{-(k+1)}}, \boldsymbol{\lambda} \in \mathbb{R}^{2} \cup i \mathbb{R}^{2}$ and $k \geq 0$. The symbols of the non-stationary butterfly scheme we construct are combinations of the symbols

$$
B_{j, h, \ell}^{[k]}\left(z_{1}, z_{2}\right)=\left(\frac{1+r_{k, 1} z_{1}}{2}\right)^{j}\left(\frac{1+r_{k, 2} z_{2}}{2}\right)^{h}\left(\frac{1+r_{k, 1} r_{k, 2} z_{1} z_{2}}{2}\right)^{\ell}, j, h, \ell \in \mathbb{N},
$$

corresponding to the three-directional exponential box splines. In this case, $M=2 I$,

$$
E=\left\{(0,0)^{T},(1,0)^{T},(0,1)^{T},(1,1)^{T}\right\}
$$

and

$$
\Xi=\left\{(1,1)^{T},(-1,1)^{T},(1,-1)^{T},(-1,-1)^{T}\right\} .
$$

It is easy to check that the symbols

$$
\begin{aligned}
a^{[k]}(\boldsymbol{z})=4\left(7 r_{k, 1} r_{k, 2} z_{1} z_{2} B_{2,2,2}^{[k]}(\boldsymbol{z})\right. & -2 r_{k, 1} z_{1} B_{1,3,3}^{[k]}(\boldsymbol{z})-2 r_{k, 2} z_{2} B_{3,1,3}^{[k]}(\boldsymbol{z}) \\
& \left.-2 r_{k, 1} r_{k, 2} z_{1} z_{2} B_{3,3,1}^{[k]}(\boldsymbol{z})\right), \quad k \geq 0,
\end{aligned}
$$

satisfy (4.23) for $\Gamma=\left\{\gamma \in \mathbb{N}_{0}^{2}:|\gamma|<4\right\}$ and for

$$
V_{k}^{\prime}=\left\{\left(-r_{k, 1}^{-1}, r_{k, 2}^{-1}\right)^{T},\left(r_{k, 1}^{-1},-r_{k, 2}^{-1}\right)^{T},\left(-r_{k, 1}^{-1},-r_{k, 2}^{-1}\right)^{T}\right\} .
$$

Thus, the scheme $S_{\left\{\mathbf{a}^{[k]}, k \geq 0\right\}}$ is $E P_{\boldsymbol{\lambda}, \Gamma}$-generating. The scheme is also interpolatory, since the symbols satisfy

$z_{1}^{-3} z_{2}^{-3}\left(a^{[k]}\left(z_{1}, z_{2}\right)+a^{[k]}\left(-z_{1}, z_{2}\right)+a^{[k]}\left(z_{1},-z_{2}\right)+a^{[k]}\left(-z_{1},-z_{2}\right)\right)=4, k \geq 0$.

Thus, by Corollary [5.1] it is also $E P_{\boldsymbol{\lambda}, \Gamma}$-reproducing with $\boldsymbol{\tau}=(0,0)^{T}$. We observe that our algebraic conditions allow us to simplify the construction of the non-stationary butterfly scheme in [29] and to improve its reproduction properties. The smoothness analysis of this scheme is done using standard techniques in [16]. 


\subsection{Non-stationary schemes obtained by convolution}

Another example is of a non-stationary subdivision scheme with dilation matrix $M=\left(\begin{array}{ll}2 & 1 \\ 0 & 2\end{array}\right)$ with $m=4$. In this case we have

$E=\left\{(0,0)^{T},(1,0)^{T},(1,1)^{T},(2,1)^{T}\right\}, \quad \Xi=\left\{(1,1)^{T},(1,-1)^{T},(-1, i)^{T},(-1,-i)^{T}\right\}$, since the coset representatives of $\mathbb{Z}^{s} / M^{T} \mathbb{Z}^{s}$ are $\left\{(0,0)^{T},(1,0)^{T},(1,1)^{T},(1,2)^{T}\right\}$. The results in [6, 14] imply that the stationary scheme associated with the symbol

$$
a(\boldsymbol{z})=\frac{1}{4} b^{2}(\boldsymbol{z}), \quad b(\boldsymbol{z})=\sum_{\boldsymbol{\varepsilon} \in E} \boldsymbol{z}^{\varepsilon},
$$

is convergent. Thus, by [15], the non-stationary scheme given by the symbols

$$
a^{[k]}(\boldsymbol{z})=\frac{1}{4}\left(b^{[k]}(\boldsymbol{z})\right)^{2}, \quad b^{[k]}(\boldsymbol{z})=\sum_{\boldsymbol{\varepsilon} \in E} \mathbf{r}_{k}^{\varepsilon} \boldsymbol{z}^{\varepsilon}, \quad k \geq 0,
$$

is also convergent, if $\mathbf{r}_{k}$ satisfy $\sum_{k \in \mathbb{Z}_{+}}\left|\mathbf{1}-\mathbf{r}_{k}\right|<\infty$. For example, we let $\mathbf{r}_{k}=$ $e^{\boldsymbol{\lambda} \cdot M^{-(k+1)}}$ for some $\boldsymbol{\lambda} \in \mathbb{R}^{2} \cup i \mathbb{R}^{2}$. The conditions in (4.15) are satisfied for the associated $V_{k}^{\prime}$. Thus, this non-stationary scheme generates polynomials $\left\{\boldsymbol{x}^{\gamma} e^{\boldsymbol{\lambda} \cdot \boldsymbol{x}}: \boldsymbol{\gamma} \in \mathbb{N}_{0}^{s},|\boldsymbol{\gamma}| \leq 1\right\}$. Furthermore, due to $q_{(0,0)}(\boldsymbol{z})=1$, we get that the condition

$$
4=a^{[k]}(\mathbf{v})=4 \cdot \mathbf{v}^{M \boldsymbol{\tau}-\boldsymbol{\tau}}, \quad \mathbf{v}=\left(r_{k, 1}^{-1}, r_{k, 2}^{-1}\right),
$$

is satisfied if and only if $\boldsymbol{\tau}=(0,0)^{T}$. Moreover, e.g. for $\boldsymbol{\gamma}=(1,0)^{T}$, the condition

$$
8=\mathbf{v}^{(1,0)} D^{(1,0)} a^{[k]}(\mathbf{v})=4 \cdot \mathbf{v}^{M \boldsymbol{\tau}-\boldsymbol{\tau}} q_{(1,0)}(\mathbf{0})=4 \cdot \mathbf{v}^{M \boldsymbol{\tau}-\boldsymbol{\tau}}
$$

cannot be satisfied for $\mathbf{v}=\left(r_{k, 1}^{-1}, r_{k, 2}^{-1}\right)$ and $\boldsymbol{\tau}=(0,0)^{T}$. Thus, we can only conclude that the non-stationary scheme reproduces $e^{\boldsymbol{\lambda} \cdot \boldsymbol{x}}$ with this shift parameter $\tau$.

We show next how to rescale the symbols $a^{[k]}(\boldsymbol{z})$ appropriately in order to enlarge the space of exponential polynomials that is being reproduced. To do so, let $a^{[k]}(\boldsymbol{z})=K^{[k]}\left(b^{[k]}(\boldsymbol{z})\right)^{2}$, where $K^{[k]}, k \geq 0$, are to be determined from our algebraic conditions. For $\mathbf{v}=\left(r_{k, 1}^{-1}, r_{k, 2}^{-1}\right)$, we get the system of equations

$$
\begin{aligned}
& 4 K^{[k]}=\mathbf{v}^{M \boldsymbol{\tau}-\boldsymbol{\tau}}, \quad M \boldsymbol{\tau}-\boldsymbol{\tau}=\left(\tau_{1}+\tau_{2}, \tau_{2}\right)^{T}, \\
& 8 K^{[k]}=\mathbf{v}^{M \boldsymbol{\tau}-\boldsymbol{\tau}} q_{(1,0)}(M \boldsymbol{\tau}-\boldsymbol{\tau})=\mathbf{v}^{M \boldsymbol{\tau}-\boldsymbol{\tau}}\left(\tau_{1}+\tau_{2}\right), \\
& 4 K^{[k]}=\mathbf{v}^{M \boldsymbol{\tau}-\boldsymbol{\tau}} q_{(0,1)}(M \boldsymbol{\tau}-\boldsymbol{\tau})=\mathbf{v}^{M \boldsymbol{\tau}-\boldsymbol{\tau}} \tau_{2} .
\end{aligned}
$$

The unique solution of this system is

$$
K^{[k]}=\frac{1}{4} \mathbf{v}^{M \boldsymbol{\tau}-\boldsymbol{\tau}} \quad \text { and } \quad \boldsymbol{\tau}=(1,1)^{T} .
$$


Thus, the non-stationary scheme given by the symbols

$$
a^{[k]}(\boldsymbol{z})=\frac{1}{4} \mathbf{r}_{k}^{-M \boldsymbol{\tau}+\boldsymbol{\tau}}\left(b^{[k]}(\boldsymbol{z})\right)^{2}, \quad b^{[k]}(\boldsymbol{z})=\sum_{\boldsymbol{\varepsilon} \in E} \mathbf{r}_{k}^{\boldsymbol{\varepsilon}} \boldsymbol{z}^{\boldsymbol{\varepsilon}}, \quad k \geq 0,
$$

generates and reproduces the space $\left\{\boldsymbol{x}^{\boldsymbol{\gamma}} e^{\boldsymbol{\lambda} \cdot \boldsymbol{x}}: \boldsymbol{\gamma} \in \mathbb{N}_{0}^{s},|\boldsymbol{\gamma}| \leq 1\right\}$ for some $\boldsymbol{\lambda} \in \mathbb{R}^{2} \cup i \mathbb{R}^{2}$ and $\boldsymbol{\tau}=(1,1)^{T}$.

\section{8. $\sqrt{3}-$ subdivision}

The results of this paper also generalize the results of [7] on polynomial reproduction of stationary schemes to the case of a general dilation matrix. This example shows how to determine the degree of polynomial reproduction of the approximating $\sqrt{3}$-subdivision schemes given in [28, page 21] from the corresponding mask symbol $a(\boldsymbol{z})$ instead of the iterated symbol $a\left(z_{1} z_{2}^{-2}, z_{1}^{2} z_{2}^{-1}\right)$. $a(\boldsymbol{z})$, as it is done in [7]. We also show how to use affine combinations of these schemes to improve their degree of polynomial reproduction, i.e. corresponding to $\Lambda=\{\mathbf{0}\}$.

The dilation matrix in this case is

$$
M=\left(\begin{array}{rr}
1 & 2 \\
-2 & -1
\end{array}\right), \quad M^{2}=-3 I,
$$

and the mask symbol is given by

$$
\begin{aligned}
a(\boldsymbol{z})= & \frac{1}{6}\left(z_{1} z_{2}+z_{1}^{-1} z_{2}^{-1}+z_{1}^{-1} z_{2}^{2}+z_{1}^{-2} z_{2}+z_{1} z_{2}^{-2}+z_{1}^{2} z_{2}^{-1}\right)+ \\
& \frac{1}{3}\left(z_{1}^{-1}+z_{2}+z_{1} z_{2}^{-1}\right)+\frac{1}{3}\left(z_{2}^{-1}+z_{1}+z_{1}^{-1} z_{2}\right) .
\end{aligned}
$$

The associated subdivision scheme satisfies zero conditions of at most order 2 , see [28]. The result of Corollary 4.8 yields $\tau=(0,0)$ in (1.2), which implies that the corresponding scheme reproduces linear polynomials. Since, the mask symbol satisfies at most zero conditions of order 2 , the associated refinable function has approximation order 2, see [30]. Note that, similarly, the corresponding $\boldsymbol{\tau}$ is $(0,0)$ for all approximating $\sqrt{3}$-subdivision schemes given in [28, page 21].

Take next the symbols $a_{j}(\boldsymbol{z}), j=1,2,3,4$ of the four approximating subdivision schemes in [28, page 21] that satisfy zero conditions of order 3 and consider their affine combination

$$
a(\boldsymbol{z})=\sum_{j=1}^{4} \lambda_{j} \cdot a_{j}(\boldsymbol{z}), \quad \sum_{j=1}^{4} \lambda_{j}=1 .
$$

The resulting scheme still satisfies Condition $\mathrm{Z}_{3}$ and in addition the rest of the conditions in Corollary 4.8 for $k=3$, if

$$
\lambda_{1}=\frac{1}{2} \lambda_{3}+\lambda_{4}+2, \quad \lambda_{2}=-\frac{3}{2} \lambda_{3}-2 \lambda_{4}-1, \quad \lambda_{3}, \lambda_{4} \in \mathbb{R} .
$$


Surprisingly, for any such choice of the parameters $\lambda_{1}, \ldots, \lambda_{4}$ the resulting scheme is given by

$a(\boldsymbol{z})=1-\frac{1}{9}\left(z_{1}^{-2}+z_{1}^{-2} z_{2}^{2}+z_{2}^{2}+z_{1}^{2}+z_{1}^{2} z_{2}^{-2}+z_{2}^{-2}\right)+\frac{4}{9}\left(z_{1}^{-1}+z_{1}^{-1} z_{2}+z_{2}+z_{1}+z_{1} z_{2}^{-1}+z_{2}^{-1}\right)$

and defines the interpolatory scheme considered in [28, page 17]. Thus, by [30], this scheme has approximation order 3 .

\section{References}

[1] C. Beccari, G. Casciola and L. Romani, A non-stationary uniform tension controlled interpolating 4-point scheme reproducing conics, Comput. Aided Geom. Design, 24 (2007), 1-9.

[2] C. Beccari, G. Casciola and L. Romani, An interpolating 4-point $C^{2}$ ternary non-stationary subdivision scheme with tension control, Comput. Aided Geom. Design, 24 (2007), 210-219.

[3] C. Beccari, G. Casciola and L. Romani, Shape-controlled interpolatory ternary subdivision, Appl. Math. Comput., 215 (2009), 916-927.

[4] C. Beccari, G. Casciola and L. Romani, A unified framework for interpolating and approximating univariate subdivision. Appl. Math. Comput., 216 (2010), 1169-1180.

[5] A. Ben-Artzi and A.Ron, Translates of exponential box splines ans their related spaces, Trans. Amer. Math. Soc. 309 (1988), 683-710.

[6] A. S. Cavaretta, W. Dahmen and C. A. Micchelli, Stationary Subdivision, Mem. Amer. Math. Soc., 453 (1991) i-vi; 1-185.

[7] M. Charina and C. Conti, Polynomial reproduction of multivariate scalar subdivision schemes, J. Comput. Applied Math., 240 (2013), 51-61,

[8] M. Charina, C. Conti, K. Jetter and G. Zimmermann, Scalar multivariate subdivision schemes and box splines, Comput. Aided Geom. Design, 28 (2011), 285-306.

[9] C. Conti and K. Hormann, Polynomial reproduction for univariate subdivision schemes of any arity, J. Approx. Theory, 163 (2011), 413-437.

[10] C. Conti, L. Gemignani and L. Romani, From approximating to interpolatory non-stationary subdivision schemes with the same generation properties, Adv. Comput. Math., 35 (2011), 217-241.

[11] C. Conti and L. Romani, Affine combination of B-spline subdivision masks and its non-stationary counterparts. BIT, 50 (2010), 269-299. 
[12] C. Conti and L. Romani, Algebraic conditions on non-stationary subdivision symbols for exponential polynomial reproduction, J. Comput. Appl. Math., 236 (2011), 543-556.

[13] C. Conti and L. Romani, Dual $m$-ary subdivision schemes of de Rham-type, Submitted for publication.

[14] S. Dahlke, W. Dahmen and V. Latour, Smooth refinable functions and wavelets obtained by convolution products, Appl. Comp. Harm. Anal., 2 (1995) 68-84.

[15] N. Dyn and D. Levin, Analysis of asymptotically equivalent binary subdivision schemes, J. Math. Anal. Appl., 193 (1995), 594-621.

[16] N. Dyn and D. Levin, Subdivision schemes in geometric modelling, Acta Numer., 11 (2002), 73-144.

[17] N. Dyn, M. Floater and K. Hormann, A $C^{2}$ four-point subdivision scheme with fourth-order accuracy and its extensions. In: M. Dæhlen, K. Mørken, L.L. Schumaker (Eds.), Mathematical Methods for Curves and Surfaces: Troms $\varnothing$ 2004, 145-156, Nashboro Press (2005).

[18] N. Dyn, D. Levin and A. Luzzatto, Exponentials Reproducing Subdivision Schemes, Found. Comput. Math. 3 (2003), 187-206.

[19] N. Dyn and A. Ron, Local approximation by certain spaces of exponential polynomials, approximation order of exponential box splines, and related intrepolation problems, Trans. Amer. Math. Soc. 319 (1990), 381-403.

[20] K. Gröchenig and A. Haas, Self-similar lattice tilings, J. Fourier Anal. Appl., 1 (1994), 131-170.

[21] B. Han, Vector cascade algorithms and refinable function vectors in Sobolev spaces, J. Approx. Theory, 124 (2003), 44-88.

[22] B. Han, Nonhomogeneous wavelet systems in high dimensions, Appl. Comp. Harm. Anal., 32 (2012), 169-196.

[23] B. Han, Properties of discrete framelet transforms, Math. Model. Nat. Phenom., in press.

[24] B. Jeong, H. O. Kim, Y. J. Lee and J. Yoon, Exponential polynomial reproducing property of non-stationary symmetric subdivision schemes and normalized exponential B-splines, Adv. Comput. Math., in press.

[25] B. Jeong, Y.J. Lee and J. Yoon, A family of non-stationary subdivision schemes reproducing exponential polynomials, J. Math. Anal. Appl., in press. 
[26] K. Jetter and G. Plonka, A survey on $L_{2}$-approximation orders from shiftinvariant spaces, in: N. Dyn, D. Leviatan, D. Levin, and A. Pinkus (eds.), Multivariate Approximation and Applications, Cambridge University Press, Cambridge, 2001, 73-111.

[27] R. Q. Jia and J. J. Lei, Approximation by piecewise exponentials, SIAM J. Math. Anal. 22 (1991), 1776-1789.

[28] Q. T. Jiang and P. Oswald, Triangular sqrt(3)-subdivision schemes: the regular case, J. Comput. Appl. Math., 156 (2003) 47-75.

[29] Y.-J. Lee, J. Yoon, Non-stationary subdivision schemes for surface interpolation based on exponential polynomials, Appl. Numer. Math., 60 (2010), 130-141.

[30] A. Levin, Polynomial generation and quasi-interpolation in stationary nonuniform subdivision, Comput. Aided Geom. Design, 20 (2003), 41-60.

[31] L. Romani, From approximating subdivision schemes for exponential splines to high-performance interpolating algorithms, J. Comput. Appl. Math. 224 (2009), 383-396.

[32] A. Ron, A necessary and sufficient condition for the linear independence of the integer translates of a compactly supported distribution, Constr. Approx. 5 (1989), 297-308.

[33] A. Ron, Exponential box splines, Constr. Approx., 4 (1988), 357-378.

[34] A. Ron, Linear independence of the translates of an exponential box spline, Rocky Mountain J. of Math., 22 (1992), 331-351.

[35] G. Strang, G.J. Fix, A Fourier analysis of the finite element variational method, in: G. Geymonat (Ed.), Constructive Aspects of Functional Analysis, in: Centro Internazionale Matematico Estivo, vol. 2, Edizioni Cremonese, Roma, 1973, 795-840.

[36] C. Vonesch, T. Blu and M. Unser, Generalized Daubechies Wavelet Families, IEEE Trans. Signal Process., 55 (2007), 4415-4429. 